\title{
Palladium-Catalyzed Carbonylation of Aryl Tosylates and Mesylates
}

\author{
Rachel H. Munday, Joseph R. Martinelli and Stephen L. Buchwald
}

\author{
Department of Chemistry, Room 18-490, \\ Massachusetts Institute of Technology, \\ Cambridge, MA 02139 (USA) \\ Fax: (+1) 617-253-3297 \\ E-mail: sbuchwal@mit.edu
}

\section{Experimental}

\section{General considerations:}

\section{Reagents}

All reactions were carried out under a carbon monoxide atmosphere. Carbon monoxide was purchased from Airgas (C.P grade, Part \# CM CP200). Pd(OAc) $)_{2}$ was purchased from Strem, Inc. or was a gift from BASF. Ligand 1, 1,3bis(dicyclohexylphosphino)propane $\left(\mathrm{dcpp} \cdot 2 \mathrm{HBF}_{4}\right)$ was received as a gift from Nippon Chemical and used as received. Anhydrous potassium phosphate was purchased from Riedel-de Haën and stored in a glovebox and potassium carbonate was purchased from $\mathrm{J}$. T. Baker and stored on the bench; both were used as received. Toluene was purchased from J. T. Baker in CYCLE-TAINER ${ }^{\circledR}$ solvent-delivery kegs and vigorously purged with argon for $2 \mathrm{~h}$, and further purified by passing the solvent two packed columns of neutral alumina and copper (II) oxide under argon pressure. Dimethyl sulfoxide, 1-butanol, triethylamine, ethanol and 1,4-dioxane were purchased from Aldrich in Sure/Seal ${ }^{\mathrm{TM}}$ bottles, used as received and stored under argon. Powdered $4 \AA$ molecular sieves were purchased from Aldrich and activated by heating with a Bunsen burner under vacuum 
prior to use. All other reagents were purchased from commercial sources and used without further purification.

\section{Analytical methods}

All new compounds and products of the carbonylation procedure were characterized by ${ }^{1} \mathrm{H}$ NMR, ${ }^{13} \mathrm{C}$ NMR, IR spectroscopy and elemental analysis. Known compounds were characterized by ${ }^{1} \mathrm{H}$ NMR and melting points (for solids) and compared to their literature values. ${ }^{1} \mathrm{H}$ and ${ }^{13} \mathrm{C}$ NMR were recorded on a Bruker $400 \mathrm{MHz}$. Infrared spectra were recorded on a Perkin-Elmer Model 2000 FT-IR using KBr plates (thin film). Elemental analyses were performed by Atlantic Microlabs Inc., Norcros, GA. All ${ }^{1} \mathrm{H}$ NMR experiments are reported in parts per million (ppm) downfield of TMS and were measured relative to the signal for chloroform $(7.27 \mathrm{ppm})$. All ${ }^{13} \mathrm{C}$ NMR spectra were reported in ppm relative to residual chloroform $(77 \mathrm{ppm})$ and were obtained with ${ }^{1} \mathrm{H}$ decoupling. Melting points were obtained on a Mel-Temp capillary melting point apparatus. Gas chromatographic analyses were performed on Hewlett-Packard 6890 gas chromatography instrument with a FID detector using $25 \mathrm{~m}$ x $0,20 \mathrm{~mm}$ capillary column with cross-linked methyl siloxane as a stationary phase.

The conversions and yields in Table 1 were determined by GC analysis using dodecane as an internal standard, added during reaction workup. The yields in Table 2 are isolated yields (average of two runs). All compounds isolated were estimated to be $\geq 95 \%$ pure as determined by ${ }^{1} \mathrm{H}$ NMR and GC analysis and/or combustion analysis. 


\section{Reaction optimization: effect of solvent and base in the Pd-catalyzed carbonylation of 4-t-butylphenyl tosylate at atmospheric pressure using ligand 1}

An oven-dried culture tube $\left(18\right.$ x $150 \mathrm{~mm}$, VWR) equipped with a Teflon ${ }^{\circledR}$-coated magnetic stir bar was charged with $4 \AA$ molecular sieves then sealed with a 14/20 rubber septum (inverted). The tube was then evacuated, heated for $\sim 1$ min with a Bunsen burner to activate the molecular sieves, cooled to room temperature then the tube was backfilled with argon. All solid reagents were added, in the air, by briefly removing the rubber septum: base ( 2 mmol, 2 equiv.), $\mathrm{Pd}(\mathrm{OAc})_{2}(2 \mathrm{~mol} \%, 0.02 \mathrm{mmol}, 0.02$ equiv., $4.5 \mathrm{mg})$ and 1,3-bis(dicyclohexylphosphino)propane $\left(\mathrm{dcpp}^{-2} \mathrm{HBF}_{4}\right)$ (4 mol \%, $0.04 \mathrm{mmol}, 0.04$ equiv.) and 4-t-butylphenyltosylate ( $1 \mathrm{mmol}, 1$ equiv., $304 \mathrm{mg}$ ). The rubber septum was then secured by wrapping with electrical tape. Next, solvent $(1 \mathrm{~mL})$ was added via syringe and the mixture stirred for $\sim 1 \mathrm{~min}$. Then $n$-butanol $(3 \mathrm{mmol}, 3$ equiv., $275 \mu \mathrm{l}$ ) was added and the mixture stirred for $\sim 5$ min. Once all reagents were added, a balloon was connected to the reaction vessel using a short length of rubber tubing ( $\sim 1$ in.), a needle adapter and a $20 \mathrm{G}$ needle. The inert atmosphere was then exchanged for carbon monoxide by briefly exposing the reaction vessel to vacuum $(\sim 1-2 \mathrm{sec})$ and backfilling with carbon monoxide; the balloon was inflated with $\mathrm{CO}_{(\mathrm{g})}$ directly following this atmosphere exchange. The reaction tube was then submerged in a preheated oil bath. The reaction mixture was heated with vigorous stirring for $15 \mathrm{~h}$. The reaction mixture was then allowed to cool to room temperature and dodecane was added as an internal standard and the reaction mixture was diluted with ethyl acetate $(\sim 10 \mathrm{~mL})$ and an aliquot was filtered through a plug of celite (eluting with ethyl acetate) and analyzed by GC. 


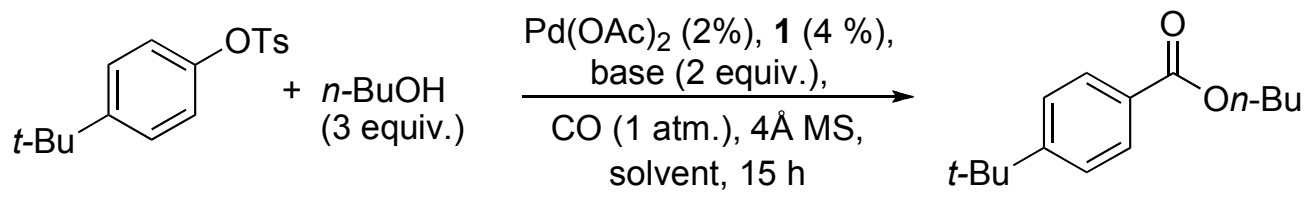

\begin{tabular}{ccccc}
\hline Entry & Base & Solvent & Temp $\left({ }^{\circ} \mathrm{C}\right)$ & Yield $(\mathbf{G C})$ \\
\hline 1 & $\mathrm{~K}_{3} \mathrm{PO}_{4}$ & toluene & 100 & 95 \\
2 & $\mathrm{~K}_{3} \mathrm{PO}_{4}$ & 1,4-dioxane & 100 & $>99$ \\
3 & $\mathrm{~K}_{3} \mathrm{PO}_{4}$ & DMSO & 100 & 90 \\
4 & $\mathrm{~K}_{3} \mathrm{PO}_{4}$ & DMSO & 90 & 98 \\
5 & $\mathrm{~K}_{3} \mathrm{PO}_{4}$ & DMSO & 80 & 32 \\
6 & $\mathrm{~K}_{3} \mathrm{PO}_{4}$ & $n$-BuOH & 100 & 92 \\
7 & $\mathrm{~K}_{2} \mathrm{CO}_{3}$ & toluene & 100 & $>99$ \\
8 & $\mathrm{~K}_{2} \mathrm{CO}_{3}$ & $1,4-$ dioxane & 100 & $>99$ \\
9 & $\mathrm{~K}_{2} \mathrm{CO}_{3}$ & DMSO & 100 & 90 \\
10 & $\mathrm{~K}_{2} \mathrm{CO}_{3}$ & $n$-BuOH & 100 & 85 \\
11 & $\mathrm{NaOAc}$ & toluene & 100 & 0 \\
\hline
\end{tabular}

\section{butyl 4-tert-butylbenzoate}

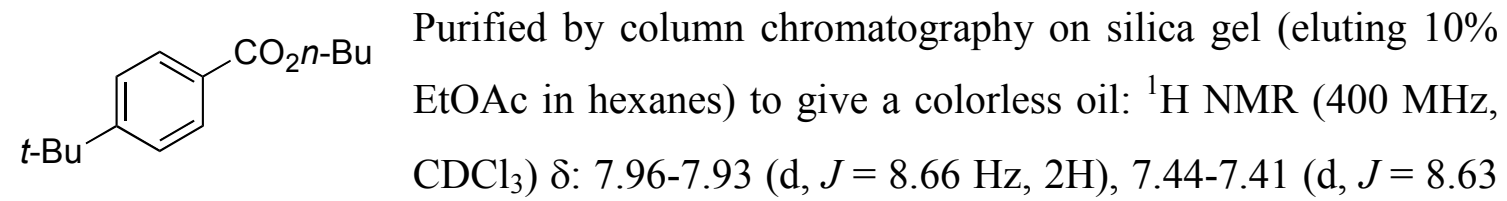

$\mathrm{Hz}, 2 \mathrm{H}), 4.30-4.27$ (t, $J=6.60 \mathrm{~Hz}, 2 \mathrm{H}), 1.75-1.68(\mathrm{~m}, 2 \mathrm{H}), 1.49-1.42$ (m, 2H), 1.31 (s, 9H), 0.97-0.93 (t, $J=7.39 \mathrm{~Hz}, 3 \mathrm{H}) .{ }^{13} \mathrm{C} \mathrm{NMR}\left(100 \mathrm{MHz}, \mathrm{CDCl}_{3}\right) \delta: 166.92,156.59$, $129.59,127.93,125.48,64.80,35.25,31.32,31.00,19.48,13.98 . \mathrm{IR}\left(\mathrm{KBr}, \mathrm{cm}^{-1}\right): 2962$, 2873, 1721, 1611, 1465, 1277, 1189, 1117, 1018, 855, 776, 708. Anal. Calcd. for $\mathrm{C}_{15} \mathrm{H}_{22} \mathrm{O}_{2}$ : C, 76.88; H, 9.46. Found: C, 76.80; H, 9.58. 


\section{Preparation of substrates: Synthesis of aryl arenesulfonates and mesylates}

\section{General procedure for the formation of aryl arenesulfonates from the corresponding phenol. ${ }^{1}$}

To a stirred solution/ suspension of phenol (1.00 equiv.) in dichloromethane $(0.5 \mathrm{M}$ concentration) cooled to $0{ }^{\circ} \mathrm{C}$ was added triethylamine (1.05 equiv.). To this was added arenesulfonyl chloride (1.05 equiv.) portionwise over $15 \mathrm{~min}$. The mixture was allowed to stir and warm gradually to room temperature overnight or until analysis by TLC showed complete consumption of starting material. The reaction was quenched with water and the phases separated. The aqueous layer was extracted with dichloromethane and the combined organics dried $\left(\mathrm{MgSO}_{4}\right)$ and concentrated in vacuo. The residue was recrystallized or purified by column chromatography on silica gel.

\section{General procedure for the formation of aryl mesylates from the corresponding phenol. $^{2}$}

To a stirred solution/suspension of phenol (1.00 equiv.) in dichloromethane (1.0 M concentration) cooled to $0{ }^{\circ} \mathrm{C}$ was added triethylamine (1.5 equiv.). To this was added mesyl chloride (1.25 equiv.) dropwise over $15 \mathrm{~min}$. The reaction was stirred at $0{ }^{\circ} \mathrm{C}$ for 15 min then quenched with water and the phases separated. The aqueous layer was extracted with dichloromethane and the combined organics dried $\left(\mathrm{MgSO}_{4}\right)$ and concentrated in vacuo. The residue was recrystallized or purified by column chromatography on silica gel.

\section{4-tert-butylphenyl 4-methylbenzenesulfonate ${ }^{3}$}

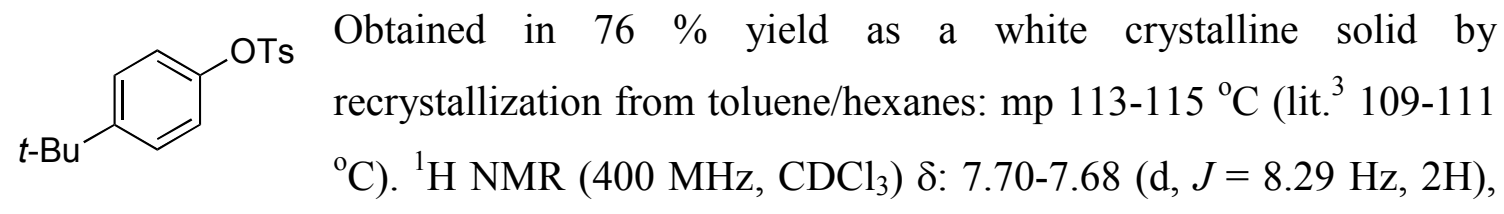

7.29-7.24 (m, 4H), 6.87-6.85 (d, $J=8.75 \mathrm{~Hz}, 2 \mathrm{H}), 2.42$ (s, 3H), 1.25 (s, 9H). ${ }^{13} \mathrm{C}$ NMR $\left(100 \mathrm{MHz}, \mathrm{CDCl}_{3}\right) \delta: 150.28,147.49,145.41,132.84,129.92,128.67,126.69,121.88$, $34.73,31.51,21.92$. 


\section{4-tert-butylphenyl methanesulfonate ${ }^{4}$}

$$
\begin{aligned}
& \text { recrystallization from methanol: } \mathrm{mp} 50-52{ }^{\circ} \mathrm{C}\left(\text { lit. }^{4} 52.5-53.5{ }^{\circ} \mathrm{C}\right) .{ }^{1} \mathrm{H} \\
& \mathrm{NMR}\left(400 \mathrm{MHz}, \mathrm{CDCl}_{3}\right) \delta: 7.41-7.37(\mathrm{~d}, J=8.87 \mathrm{~Hz}, 2 \mathrm{H}), 7.19-
\end{aligned}
$$

$7.15(\mathrm{~d}, J=8.87 \mathrm{~Hz}, 2 \mathrm{H}), 3.08(\mathrm{~s}, 3 \mathrm{H}), 1.28(\mathrm{~s}, 9 \mathrm{H}) .{ }^{13} \mathrm{C} \mathrm{NMR}\left(100 \mathrm{MHz}, \mathrm{CDCl}_{3}\right) \delta$ : $150.70,147.18,127.14,121.60,37.34,34.81,31.52$.

\section{4-tert-butylphenyl benzenesulfonate}

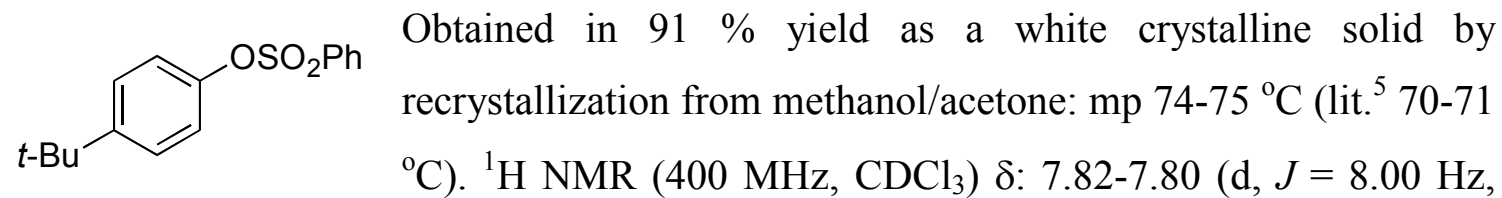

2H), 7.65-7.61 (t, $J=7.49 \mathrm{~Hz}, 1 \mathrm{H}), 7.51-7.47(\mathrm{t}, J=7.91 \mathrm{~Hz}, 2 \mathrm{H}), 7.27-7.23(\mathrm{~d}, J=8.76$

$\mathrm{Hz}, 2 \mathrm{H}), 6.87-6.84$ (d, $J=8.74 \mathrm{~Hz}, 2 \mathrm{H}), 1.24$ (s, 9H). ${ }^{13} \mathrm{C} \mathrm{NMR}\left(100 \mathrm{MHz}, \mathrm{CDCl}_{3}\right) \delta$ : $150.41,147.42,135.76,134.38,129.33,128.64,126.75,121.84,34.74,31.50$.

\section{pyridin-3-yl 4-methylbenzenesulfonate ${ }^{6}$}

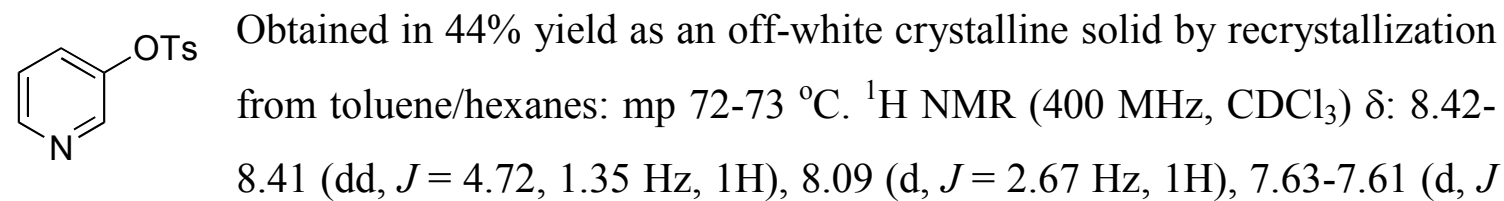
$=8.36 \mathrm{~Hz}, 2 \mathrm{H}), 7.38-7.35(\mathrm{ddd}, J=8.39,2.71,1.42 \mathrm{~Hz}, 1 \mathrm{H}), 7.27-7.25(\mathrm{~d}, J=8.08 \mathrm{~Hz}$, 2H), 7.23-7.20 (dd, $J=8.36,4.70 \mathrm{~Hz}, 1 \mathrm{H}), 2.37$ (s, 3H). ${ }^{13} \mathrm{C} \mathrm{NMR}\left(100 \mathrm{MHz}, \mathrm{CDCl}_{3}\right) \delta$ : $148.40,146.60,146.27,144.16,131.68,130.37,130.23,128.66,128.31,124.42,21.92$. IR (KBr, cm $\left.{ }^{-1}\right): 3090,1595,1476,1423,1368,1200,1186,1091,858,823,747$.

\section{benzo[d][1,3]dioxol-5-yl 4-methylbenzenesulfonate}

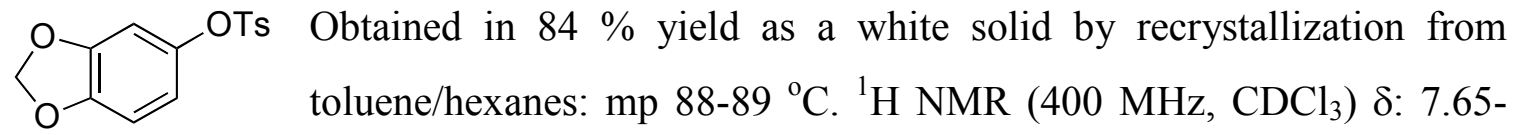
$7.63(\mathrm{~d}, J=8.31 \mathrm{~Hz}, 2 \mathrm{H}), 7.27-7.25(\mathrm{~d}, J=8.47 \mathrm{~Hz}, 2 \mathrm{H}), 6.58-6.56(\mathrm{~d}, J=8.46 \mathrm{~Hz}, 1 \mathrm{H})$, 6.47-6.46 (d, $J=2.39 \mathrm{~Hz}, 1 \mathrm{H}), 6.32-6.29(\mathrm{dd}, J=8.46,2.40 \mathrm{~Hz}, 1 \mathrm{H}), 5.89$ (s, 2H), 2.38 
(s, 3H). $\left.{ }^{13} \mathrm{C} \mathrm{NMR} \mathrm{(100} \mathrm{MHz,} \mathrm{CDCl}_{3}\right) \delta: 148.16,146.60,145.72,143.90,132.23,130.00$, $128.70,115.37,108.01,104.55,102.20,21.89$.

\section{2-methylbenzo $[d]$ thiazol-5-yl methanesulfonate}

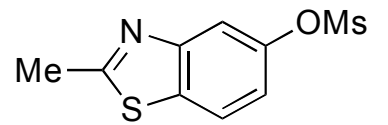

Obtained in $72 \%$ yield as an off-white solid by recrystallization from toluene/hexanes: mp 110-112 ${ }^{\circ} \mathrm{C} .{ }^{1} \mathrm{H}$ NMR $(400 \mathrm{MHz}$, $\left.\mathrm{CDCl}_{3}\right)$ \&: 7.80-7.79 (d, $\left.J=2.36 \mathrm{~Hz}, 1 \mathrm{H}\right), 7.79-7.77(\mathrm{~d}, J=8.84 \mathrm{~Hz}, 1 \mathrm{H}), 7.28-7.25(\mathrm{dd}, J$ $=8.69,2.35 \mathrm{~Hz}, 1 \mathrm{H}), 3.13(\mathrm{~s}, 3 \mathrm{H}), 2.79(\mathrm{~s}, 3 \mathrm{H}) \cdot{ }^{13} \mathrm{C} \mathrm{NMR}\left(100 \mathrm{MHz}, \mathrm{CDCl}_{3}\right) \delta: 170.11$, $154.19,147.82,134.84,122.53,119.41,115.86,37.49,20.50$. IR $\left(\mathrm{KBr}, \mathrm{cm}^{-1}\right): 3058$, 1562, 1518, 1451, 1351, 1175, 1121, 986, 947, 782, 614. Anal. Calcd. for $\mathrm{C}_{9} \mathrm{H}_{9} \mathrm{NO}_{3} \mathrm{~S}_{2}: \mathrm{C}$, 44.43; H, 3.73. Found: C, 44.51; H, 3.78.

\section{4-(1H-pyrrol-1-yl)phenyl methanesulfonate}

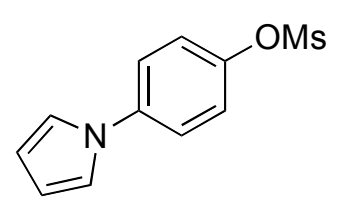

Obtained in $81 \%$ yield as a white solid by column chromatography on silica gel (gradient elution: 20-50\% EtOAc in hexanes): $\mathrm{mp}$ 135-136 ${ }^{\circ} \mathrm{C} .{ }^{1} \mathrm{H}$ NMR $\left(400 \mathrm{MHz}, \mathrm{CDCl}_{3}\right) \delta: 7.46-7.42(\mathrm{~d}, J=9.10$ $\mathrm{Hz}, 2 \mathrm{H}), 7.38-7.35$ (d, $J=9.13 \mathrm{~Hz}, 2 \mathrm{H}), 7.08-7.07$ (t, $J=2.20 \mathrm{~Hz}, 2 \mathrm{H}), 6.39-6.38$ (t, $J=$ $2.19 \mathrm{~Hz}, 2 \mathrm{H}), 3.19$ (s, 3H). ${ }^{13} \mathrm{C}$ NMR $\left(100 \mathrm{MHz}, \mathrm{CDCl}_{3}\right) \delta: 146.60,139.99,123.51$, 121.91, 119.59, 111.26, 37.62. IR ( $\left.\mathrm{KBr}, \mathrm{cm}^{-1}\right): 3145,3038,1520,1376,1181,1162$, 1114, 972, 868, 841, 727. ${ }^{13} \mathrm{C}$ NMR: $\delta$ 146.60, 139.99, 123.51, 121.91, 119.59, 111.26, 37.62. Anal. Calcd. for $\mathrm{C}_{11} \mathrm{H}_{11} \mathrm{NO}_{3} \mathrm{~S}: \mathrm{C}, 55.68 ; \mathrm{H}, 4.67$. Found: C, 55.69; H, 4.70.

\section{4-ethanoyl-2-methoxyphenyl methanesulfonate}

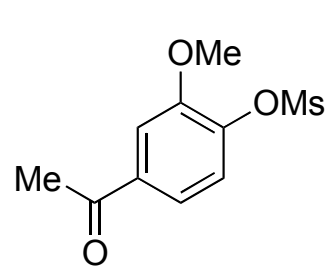

Obtained in $96 \%$ yield as a white solid by column chromatography on silica gel (50\% EtOAc in hexanes): $\mathrm{mp} 80-82{ }^{\circ} \mathrm{C} .{ }^{1} \mathrm{H}$ NMR (400 $\left.\mathrm{MHz}, \mathrm{CDCl}_{3}\right) \delta: 7.55(\mathrm{~d}, J=1.94 \mathrm{~Hz}, 1 \mathrm{H}), 7.50-7.48(\mathrm{dd}, J=8.29$, $1.97 \mathrm{~Hz}, 1 \mathrm{H}), 7.31-7.29$ (d, $J=8.28 \mathrm{~Hz}, 1 \mathrm{H}), 3.88$ (s, 3H), 3.16 (s,

$3 \mathrm{H}), 2.53(\mathrm{~s}, 3 \mathrm{H}) .{ }^{13} \mathrm{C}$ NMR (100 MHz, $\left.\mathrm{CDCl}_{3}\right) \delta: 196.88,151.83,141.95,137.01$, 124.62, 122.20, 112.07, 56.37, 38.86, 26.79. IR ( $\left.\mathrm{KBr}, \mathrm{cm}^{-1}\right): 3038,2937,1691,1598$, 1361, 1163, 1115, 1026, 977, 853, 791. Anal. Calcd. for $\mathrm{C}_{10} \mathrm{H}_{12} \mathrm{O}_{5} \mathrm{~S}: \mathrm{C}, 49.17 ; \mathrm{H}, 4.95$. Found: C, 49.00; H, 4.86 . 


\section{5-formyl-2-methoxyphenyl 4-methylbenzenesulfonate ${ }^{7}$}

OHC OTs Obtained in $97 \%$ yield as white crystals by recrystallization from toluene/hexanes: mp $135-137{ }^{\circ} \mathrm{C}$ (lit. $\left.{ }^{7} 136-138{ }^{\circ} \mathrm{C}\right) .{ }^{1} \mathrm{H}$ NMR (400 $\left.\mathrm{MHz}, \mathrm{CDCl}_{3}\right) \delta: 9.75(\mathrm{~s}, 1 \mathrm{H}), 7.71-7.66(\mathrm{~m}, 3 \mathrm{H}), 7.58(\mathrm{~d}, J=1.98$ $\mathrm{Hz}, 1 \mathrm{H}), 7.27-7.25$ (d, $J=8.01 \mathrm{~Hz}, 2 \mathrm{H}), 6.93-6.91(\mathrm{~d}, J=8.51 \mathrm{~Hz}, 1 \mathrm{H}), 3.60$ (s, 3H), 2.38 (s, 3H). ${ }^{13} \mathrm{C}$ NMR (100 MHz, $\left.\mathrm{CDCl}_{3}\right) \delta: 189.90,157.09,145.77,138.89,132.92$, $130.86,129.86,129.76,128.69,124.88,112.72,56.21,21.87 . \mathrm{IR}\left(\mathrm{KBr}, \mathrm{cm}^{-1}\right)$ : 1692 , 1597, 1363, 1180, 1103, 1086, 951, 812, 726, 668. Anal. Calcd. for $\mathrm{C}_{15} \mathrm{H}_{14} \mathrm{O}_{5} \mathrm{~S} ; \mathrm{C}, 58.81$; H, 4.61. Found: C, 58.65; H, 4.63.

\section{2,4-dimethylphenyl 4-methylbenzenesulfonate ${ }^{8}$}

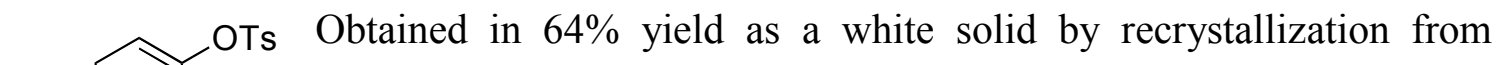
toluene/hexanes: mp 69-70 ${ }^{\circ} \mathrm{C}$ (lit. $71{ }^{\circ} \mathrm{C}$ ). ${ }^{1} \mathrm{H}$ NMR $(400 \mathrm{MHz}$, $\left.\mathrm{CDCl}_{3}\right) \delta: 7.70-7.68(\mathrm{~d}, J=8.34 \mathrm{~Hz}, 2 \mathrm{H}), 7.28-7.26(\mathrm{~d}, J=8.00 \mathrm{~Hz}$,

2H), 6.91-6.80 (m, 3H), $2.41(\mathrm{~s}, 3 \mathrm{H}), 2.23(\mathrm{~s}, 3 \mathrm{H}), 2.00(\mathrm{~s}, 3 \mathrm{H}) .{ }^{13} \mathrm{C}$ NMR $(100 \mathrm{MHz}$, $\left.\mathrm{CDCl}_{3}\right) \delta: 146.38,145.52,136.94,133.34,132.36,131.32,130.00,128.57,127.63$, $122.16,21.89,20.99,16.42$.

\section{3-(trifluoromethyl)phenyl methanesulfonate ${ }^{4}$}

$\mathrm{F}_{3} \mathrm{C}$ OMs Obtained in $92 \%$ yield as a colorless oil by column chromatography on silica gel (eluting 30\% EtOAc in hexanes): ${ }^{1} \mathrm{H}$ NMR (400 MHz, $\left.\mathrm{CDCl}_{3}\right) \delta: 7.59-7.46(\mathrm{~m}, 4 \mathrm{H}), 3.17(\mathrm{~s}, 3 \mathrm{H}) .{ }^{13} \mathrm{C} \mathrm{NMR}\left(100 \mathrm{MHz}, \mathrm{CDCl}_{3}\right) \delta: 149.17$, $132.77\left(\mathrm{q}, J_{\mathrm{CF}}=33.45 \mathrm{~Hz}\right.$, chemical shifts for four peaks 132.27, 132.93, 132.60, 132.27), $130.96,125.88,124.43\left(\mathrm{q}, J_{\mathrm{CF}}=3.72 \mathrm{~Hz}\right.$, chemical shifts for four peaks 124.49 , $124.45,124.42,124.38), 123.34\left(\mathrm{q}, J_{\mathrm{CF}}=272.6 \mathrm{~Hz}\right.$, chemical shifts for four peaks $127.41,124.70,121.99,119.28), 119.51\left(\mathrm{q}, J_{\mathrm{CF}}=3.83 \mathrm{~Hz}\right.$, chemical shifts for four peaks 119.57, 119.53, 119.49, 119.45), 37.97. IR (KBr, cm $\left.{ }^{-1}\right): 3083,3036,2944,1595,1493$, 1450, 1380, 1327, 1280, 1127, 1092, 1067, 909, 810. Anal. Calcd. for $\mathrm{C}_{8} \mathrm{H}_{7} \mathrm{~F}_{3} \mathrm{O}_{3} \mathrm{~S}: \mathrm{C}$, 40.00; H, 2.94. Found: C, 40.21; H, 2.93. 


\section{3-(trifluoromethyl)phenyl 4-methylbenzenesulfonate ${ }^{9}$}

OTs Obtained in $65 \%$ yield as a white solid by recrystallization from toluene/hexanes: mp 58-60 ${ }^{\circ} \mathrm{C}$ (lit. $\left.{ }^{9} 62-64{ }^{\circ} \mathrm{C}\right) .{ }^{1} \mathrm{H}$ NMR $(400 \mathrm{MHz}$, $\left.\mathrm{CDCl}_{3}\right) \delta: 7.68-7.66(\mathrm{~d}, J=8.36 \mathrm{~Hz}, 2 \mathrm{H}), 7.50-7.48(\mathrm{~d}, J=7.81 \mathrm{~Hz}, 1 \mathrm{H}), 7.43-7.39$ (t, $J$ $=7.97 \mathrm{~Hz}, 1 \mathrm{H}), 7.31-7.29(\mathrm{~d}, J=8.33 \mathrm{~Hz}, 2 \mathrm{H}), 7.20-7.15(\mathrm{~m}, 2 \mathrm{H}), 2.43(\mathrm{~s}, 3 \mathrm{H}) .{ }^{13} \mathrm{C}$ NMR $\left(100 \mathrm{MHz}, \mathrm{CDCl}_{3}\right) \delta: 149.77,146.16,132.32$ (q, $J=33.28 \mathrm{~Hz}$, chemical shifts for 4 peaks: $132.81,132.48,132.15,131.82), 131.98,130.52,130.12,128.71,126.22,124.09$ (q, $J=3.72 \mathrm{~Hz}$, chemical shifts for 4 peaks: 124.15, 124.11, 124.07, 124.04), 123.31 (q, $J$ $=272.55 \mathrm{~Hz}$, chemical shifts for 4 peaks: 127.37, 124.66, 121.95, 119.24), 119.94 (q, $J=$ $3.85 \mathrm{~Hz}$, chemical shifts for 4 peaks: 120.00, 119.96, 119.92, 119.88), 21.90. IR (KBr, $\mathrm{cm}^{-1}$ ): 3090, 2927, 1595, 1448, 1353, 1327, 1197, 1164, 1133, 1089, 1063, 893, 807, 769, 730, 703, 554. Anal. Calcd. for $\mathrm{C}_{14} \mathrm{H}_{11} \mathrm{~F}_{3} \mathrm{O}_{3} \mathrm{~S}: \mathrm{C}$, 53.16; H, 3.51. Found: C, 53.23; H, 3.41 .

\section{2-methylquinolin-8-yl 4-methylbenzenesulfonate}

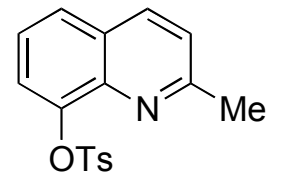

Obtained in $53 \%$ yield as a white crystalline solid by recrystallization from toluene/hexanes: mp 125-127 ${ }^{\circ} \mathrm{C} .{ }^{1} \mathrm{H}$ NMR (400 MHz, $\left.\mathrm{CDCl}_{3}\right) \delta$ : 7.88-7.86 (d, $J=8.45 \mathrm{~Hz}, 1 \mathrm{H}), 7.80-7.78(\mathrm{~d}, J=8.33 \mathrm{~Hz}, 2 \mathrm{H}), 7.60-$ $7.58(\mathrm{dd}, J=8.17,1.13 \mathrm{~Hz}, 1 \mathrm{H}), 7.57-7.54$ (dd, $J=7.67,1.22 \mathrm{~Hz}, 1 \mathrm{H}), 7.36-7.32$ (t, $J=$ $7.91 \mathrm{~Hz}, 1 \mathrm{H}), 7.15-7.11(\mathrm{~m}, 3 \mathrm{H}), 2.45$ (s, 3H), 2.29 (s, 3H). ${ }^{13} \mathrm{C} \mathrm{NMR}\left(100 \mathrm{MHz}, \mathrm{CDCl}_{3}\right)$ $\delta: 159.73,145.14,145.09,141.03,135.95,133.54,129.37,129.05,128.04,127.02$, 125.23, 123.19, 122.88, 25.41, 21.77. IR ( $\left.\mathrm{KBr}, \mathrm{cm}^{-1}\right): 3062,2952,2921,1598,1432$, 1367, 1161, 1070, 909, 820, 783. Anal. Calcd. for $\mathrm{C}_{17} \mathrm{H}_{15} \mathrm{NO}_{3} \mathrm{~S}$ : C, 65.16; H, 4.82 . Found: C, 65.27; H, 4.81.

\section{3,4,5-trimethoxyphenyl methanesulfonate ${ }^{10}$}

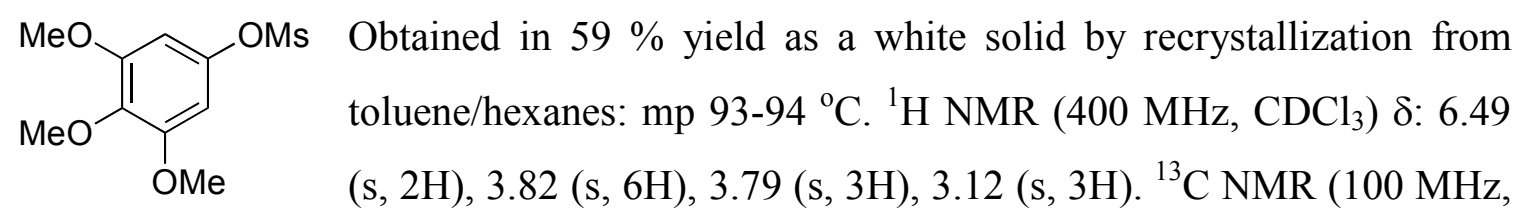

$\left.\mathrm{CDCl}_{3}\right) \delta: 153.88,145.20,137.19,99.70,61.14,56.51,37.53 . \mathrm{IR}\left(\mathrm{KBr}, \mathrm{cm}^{-1}\right): 3025$, 2934, 1608, 1468, 1422, 1367, 1229, 1128, 1003, 988, 967, 853. 


\section{3-cyanophenyl 4-methylbenzenesulfonate}

NC OTs Obtained in $50 \%$ yield as a white solid by recrystallization from toluene/hexanes: mp 85-87 ${ }^{\circ} \mathrm{C} .{ }^{1} \mathrm{H}$ NMR (400 MHz, $\left.\mathrm{CDCl}_{3}\right) \delta$ : 7.68$7.66(\mathrm{~d}, J=8.30 \mathrm{~Hz}, 2 \mathrm{H}), 7.53-7.51(\mathrm{~d}, J=7.72 \mathrm{~Hz}, 1 \mathrm{H}), 7.43-7.39$ (t, $J=8.02 \mathrm{~Hz}, 1 \mathrm{H}), 7.33-7.31(\mathrm{~d}, J=8.08 \mathrm{~Hz}, 2 \mathrm{H}), 7.28-7.25(\mathrm{~m}, 1 \mathrm{H}), 7.22-7.21(\mathrm{~m}, 1 \mathrm{H})$, 2.44 (s, 3H). ${ }^{13} \mathrm{C}$ NMR (100 MHz, $\left.\mathrm{CDCl}_{3}\right) \delta: 149.76,146.41,131.78,131.03,130.97$, 130.30, 128.66, 127.67, 126.20, 117.53, 113.88, 21.99. IR (KBr, $\left.\mathrm{cm}^{-1}\right): 3083,2231,1580$, 1381, 1191, 1177, 1090, 934, 808, 770, 680, 548. Anal. Calcd. for $\mathrm{C}_{14} \mathrm{H}_{11} \mathrm{NO}_{3} \mathrm{~S}: \mathrm{C}, 61.52$; H, 4.06. Found: C, 61.30; H, 4.12.

\section{biphenyl-4-yl methanesulfonate ${ }^{10}$}

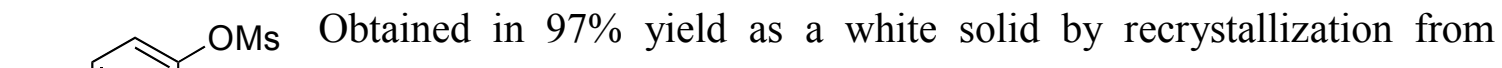
toluene/hexanes: mp 152-153 ${ }^{\circ} \mathrm{C}$ (lit. ${ }^{4} 151-152{ }^{\circ} \mathrm{C}$ ). ${ }^{1} \mathrm{H}$ NMR (400 $\left.\mathrm{MHz}, \mathrm{CDCl}_{3}\right) \delta: 7.62-7.58(\mathrm{~d}, J=8.84 \mathrm{~Hz}, 2 \mathrm{H}), 7.55-7.52(\mathrm{~m}, 2 \mathrm{H})$, 7.45-7.41 (m, 2H), 7.38-7.32 (m, 1H), 7.35-7.33 (d, $J=8.83 \mathrm{~Hz}, 2 \mathrm{H}), 3.15(\mathrm{~s}, 3 \mathrm{H}) .{ }^{13} \mathrm{C}$ NMR (100 MHz, $\left.\mathrm{CDCl}_{3}\right) \delta: 148.75,140.86,139.93,129.13,128.92,127.98,127.35$, 122.52, 37.59. IR (KBr, $\left.\mathrm{cm}^{-1}\right): 2942,1486,1366,1190,1175,1153,848,765$.

\section{3-(dimethylanimo)phenyl methanesulfonate ${ }^{2}$}

$\mathrm{Me}_{2} \mathrm{~N}$ OMs Obtained in $91 \%$ yield as a slightly pink solid by flash column chromatography (gradient elution: $0 \%$ to $10 \%$ to $20 \%$ EtOAc in

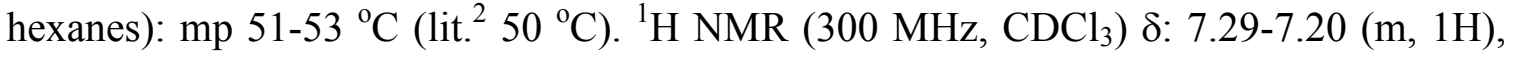
6.68-6.56 (m, 3H), $3.14(\mathrm{~s}, 3 \mathrm{H}), 2.98(\mathrm{~s}, 6 \mathrm{H})$.

\section{4-methoxyphenyl 4-fluorobenzenesulfonate ${ }^{11}$}

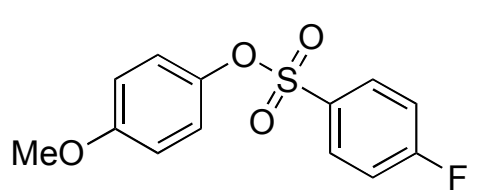

Obtained in $81 \%$ yield as a white solid by recrystallization from toluene/hexanes: $\mathrm{mp} 68-69{ }^{\circ} \mathrm{C}$ (lit. ${ }^{11}$ 66-67 $\left.{ }^{\circ} \mathrm{C}\right) .{ }^{1} \mathrm{H}$ NMR (400 MHz, $\left.\mathrm{CDCl}_{3}\right) \delta: 7.84-7.81(\mathrm{~m}$, 2H), 7.22-7.17 (t, $J=8.47 \mathrm{~Hz}, 2 \mathrm{H}), 6.89-6.87(\mathrm{~d}, J=9.12 \mathrm{~Hz}, 2 \mathrm{H}), 6.79-6.77(\mathrm{~d}, J=9.02$ $\mathrm{Hz}, 2 \mathrm{H}), 3.76(\mathrm{~s}, 3 \mathrm{H}) .{ }^{13} \mathrm{C} \mathrm{NMR}\left(100 \mathrm{MHz}, \mathrm{CDCl}_{3}\right) \delta: 166.15(\mathrm{~d}, J=257.22 \mathrm{~Hz}$, chemical shifts of 2 peaks: 167.43, 164.87), 158.53, 143.01, 131.62 (d, $J=9.65 \mathrm{~Hz}$, 
chemical shifts of 2 peaks: $131.67,131.58), 131.38(\mathrm{~d}, J=3.24 \mathrm{~Hz}$, chemical shifts of 2 peaks: $131.40,131.37), 123.47,116.71(\mathrm{~d}, J=22.78 \mathrm{~Hz}$, chemical shifts of 2 peaks: 116.82, 116.60), 114.76, 55.74. IR (KBr, $\left.\mathrm{cm}^{-1}\right): 3107,3070,2845,1590,1496,1373$, 1238, 1195, 1175, 1153, 1031, 840. Anal. Calcd. for $\mathrm{C}_{13} \mathrm{H}_{11} \mathrm{FO}_{4} \mathrm{~S}: \mathrm{C}, 55.31 ; \mathrm{H}, 3.93$. Found: C, 55.35; H, 3.76 .

\section{5,6,7,8-tetrahydronaphthalen-2-yl 4-fluorobenzenesulfonate}

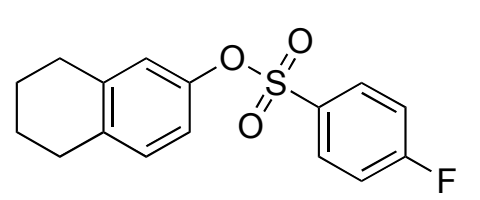

Obtained in $52 \%$ yield as a white solid by recrystallization from toluene/hexanes: mp 58-60 ${ }^{\circ} \mathrm{C} .{ }^{1} \mathrm{H}$ NMR $(400 \mathrm{MHz}$, $\left.\mathrm{CDCl}_{3}\right) \delta: 7.85-7.81(\mathrm{~m}, 2 \mathrm{H}), 7.23-7.15(\mathrm{t}, J=8.35 \mathrm{~Hz}$, 2H), 6.92-6.90 (d, $J=8.36 \mathrm{~Hz}, 1 \mathrm{H}), 6.69-6.68(\mathrm{~d}, J=1.97 \mathrm{~Hz}, 1 \mathrm{H}), 6.61-6.58$ (dd, $J=$ 8.34, $2.26 \mathrm{~Hz}, 1 \mathrm{H}), 2.67-2.65(\mathrm{~m}, 4 \mathrm{H}), 1.74-1.71(\mathrm{~m}, 4 \mathrm{H}) .{ }^{13} \mathrm{C} \mathrm{NMR}\left(100 \mathrm{MHz}, \mathrm{CDCl}_{3}\right)$ $\delta: 166.11$ (d, $J=257.07 \mathrm{~Hz}$, chemical shifts of 2 peaks: 167.38, 164.83), 147.22, 139.12, $136.58,131.81$ (d, $J=3.28 \mathrm{~Hz}$, chemical shifts of 2 peaks: 131.83, 131.79), 131.57 (d, $J$ $=9.66 \mathrm{~Hz}$, chemical shifts of 2 peaks: 131.62, 131.53), 130.31, 122.63, 119.21, 116.64 (d, $J=22.79 \mathrm{~Hz}$, chemical shifts of 2 peaks: 116.75, 116.53), 29.53, 29.04, 23.05, 22.83.

IR $\left(\mathrm{KBr}, \mathrm{cm}^{-1}\right): 3108,3074,2934,2860,1593,1494,1378,1237,1215,1194,1183$, $1158,1136,1093,925,839$, 808. Anal. Calcd. for $\mathrm{C}_{16} \mathrm{H}_{15} \mathrm{FO}_{3} \mathrm{~S}: \mathrm{C}, 62.73 ; \mathrm{H}, 4.94$. Found: C, 62.84; H, 4.93. 
General Procedure: Pd-Catalyzed Carbonylation of Aryl Arenesulfonates at Atmospheric Pressure

An oven-dried culture tube $\left(18\right.$ x $150 \mathrm{~mm}$, VWR) equipped with a Teflon ${ }^{\circledR}$ coated magnetic stir bar was charged with $4 \AA$ molecular sieves then sealed with a $14 / 20$ rubber septum (inverted). The tube was then evacuated, heated for $\sim 1$ min with a Bunsen burner to activate the molecular sieves, then the tube was backfilled with argon and allowed to cool under argon. All solid reagents were added, in the air, by briefly removing the rubber septum: $\mathrm{K}_{2} \mathrm{CO}_{3}$ (2 equiv., $\left.276 \mathrm{mg}\right), \mathrm{Pd}(\mathrm{OAc})_{2}(2 \mathrm{~mol} \%, 0.02 \mathrm{mmol}, 0.02$ equiv., $4.5 \mathrm{mg})$ and 1,3-bis(dicyclohexylphosphino)propane (dcpp $\left.2 \mathrm{HBF}_{4}\right)(2.2 \mathrm{~mol} \%, 0.022$ mmol, 0.022 equiv., $14.0 \mathrm{mg}$ ) and aryl arenesulfonate/mesylate (1 mmol, 1 equiv.). After the addition of all solid reagents, the rubber septum was secured by wrapping with electrical tape. Next, toluene $(1 \mathrm{~mL})$ was added via syringe and the mixture stirred for $1 \mathrm{~min}$. Alcohol ( $3 \mathrm{mmol}, 3$ equiv.) was then added and the mixture stirred for $\sim 5$ min (Alcohols which were solids at room temperature were added during the initial charge). Once all reagents were added, a balloon was connected to the reaction vessel using a short length of rubber tubing ( $1 \mathrm{in}$.), a needle adapter and a $20 \mathrm{G}$ needle. The inert atmosphere was then exchanged for carbon monoxide by briefly exposing the reaction vessel to vacuum ( 1-2 sec) and backfilling with carbon monoxide; the balloon was inflated with $\mathrm{CO}_{(\mathrm{g})}$ directly following this atmosphere exchange. The reaction tube was then submerged in a preheated oil bath $\left(80-110{ }^{\circ} \mathrm{C}\right)$. The reaction mixture was heated with vigorous stirring for $15 \mathrm{~h}$. The reaction mixture was then allowed to cool to room temperature, diluted with methylene chloride or ethyl acetate $(\sim 10 \mathrm{~mL})$, filtered through a plug of celite (eluting with methylene chloride or ethyl acetate) and concentrated under reduced pressure. The crude material obtained was purified by flash chromatography on silica gel.

\section{Butyl pyridine-3-carboxylate ${ }^{12}$}

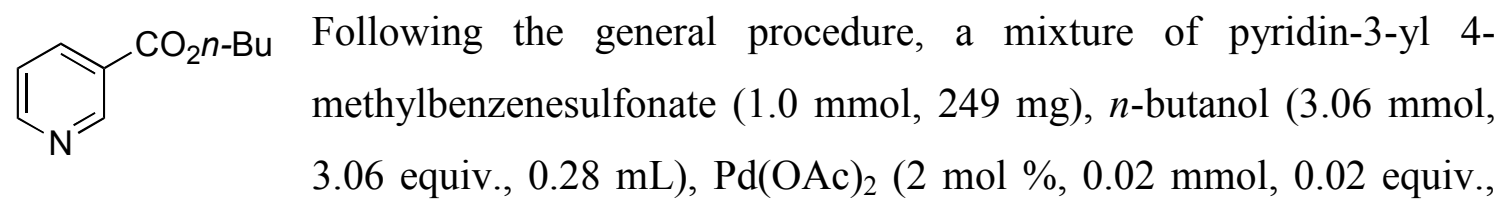

$4.5 \mathrm{mg})$, depp $2 \mathrm{HBF}_{4}(2.2 \mathrm{~mol} \%, 0.022 \mathrm{mmol}, 0.022$ equiv., $14.0 \mathrm{mg}), \mathrm{K}_{2} \mathrm{CO}_{3}(2.0$ 
mmol, 2.0 equiv., $276 \mathrm{mg}), 4 \AA$ molecular sieves $(150 \mathrm{mg})$, and toluene $(1.0 \mathrm{~mL})$ was heated at $90{ }^{\circ} \mathrm{C}$ for $15 \mathrm{~h}$. The reaction mixture was filtered through Celite eluting with ethyl acetate and the solvent was removed in vacuo. The residue was purified by flash chromatography on silica gel (20\% EtOAc in hexanes) to provide the title compound as a colorless oil (150 mg, $84 \%)$. ${ }^{1} \mathrm{H}$ NMR $\left(400 \mathrm{MHz}, \mathrm{CDCl}_{3}\right) \delta$ : 9.18-9.17 (d, $J=1.16 \mathrm{~Hz}$, $1 \mathrm{H}), 8.72-8.71(\mathrm{~d}, J=4.83 \mathrm{~Hz}, 1 \mathrm{H}), 8.26-8.23(\mathrm{~d}, J=7.96 \mathrm{~Hz}, 1 \mathrm{H}), 7.35-7.32$ (dd, $J=$ $7.92,4.87 \mathrm{~Hz}, 1 \mathrm{H}), 4.32-4.28$ (t, $J=6.63 \mathrm{~Hz}, 2 \mathrm{H}), 1.74-1.67(\mathrm{~m}, 2 \mathrm{H}), 1.47-1.38(\mathrm{~m}, 2 \mathrm{H})$, 0.94-0.91 (t, $J=7.39 \mathrm{~Hz}, 3 \mathrm{H}) .{ }^{13} \mathrm{C}$ NMR $\left(100 \mathrm{MHz}, \mathrm{CDCl}_{3}\right) \delta: 165.49,153.46,151.03$, 137.21, 126.51, 123.45, 65.47, 30.83, 19.39, 13.91. IR (KBr, $\left.\mathrm{cm}^{-1}\right): 2961,1725,1591$, 1420, 1285, 1113, 1025, 742. Anal. Calcd. for $\mathrm{C}_{10} \mathrm{H}_{13} \mathrm{NO}_{2}$ : C, 67.02; H, 7.31. Found: $\mathrm{C}$, $66.98 ; \mathrm{H}, 7.48$.

\section{butyl benzo[ $[d][1,3]$ dioxole-5-carboxylate ${ }^{13}$}

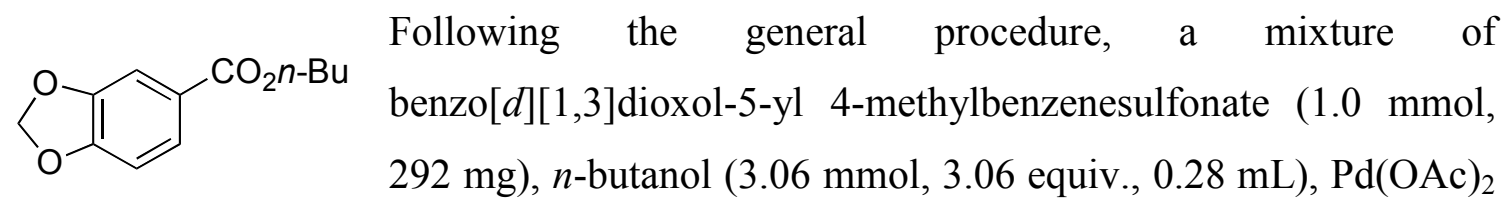

( $2 \mathrm{~mol} \%, 0.02 \mathrm{mmol}, 0.02$ equiv., $4.5 \mathrm{mg}$ ), dcpp• $2 \mathrm{HBF}_{4}(2.2 \mathrm{~mol} \%, 0.022 \mathrm{mmol}, 0.022$ equiv., $14.0 \mathrm{mg}$ ), $\mathrm{K}_{2} \mathrm{CO}_{3}(2.0 \mathrm{mmol}, 2.0$ equiv., $276 \mathrm{mg}), 4 \AA$ molecular sieves $(150 \mathrm{mg})$, and toluene $(1.0 \mathrm{~mL})$ was heated at $100{ }^{\circ} \mathrm{C}$ for $15 \mathrm{~h}$. The reaction mixture was filtered through Celite eluting with ethyl acetate and the solvent was removed in vacuo. The residue was purified by flash chromatography on silica gel (10\% EtOAc in hexanes) to provide the title compound as a colorless oil $(201 \mathrm{mg}, 91 \%) .{ }^{1} \mathrm{H}$ NMR $(400 \mathrm{MHz}$, $\left.\mathrm{CDCl}_{3}\right) \delta$ : 7.67-7.64 (dd, $\left.J=8.18,1.66 \mathrm{~Hz}, 1 \mathrm{H}\right), 7.47(\mathrm{~d}, J=1.65 \mathrm{~Hz}, 1 \mathrm{H}), 6.85-6.83(\mathrm{~d}$, $J=8.17 \mathrm{~Hz}, 1 \mathrm{H}), 6.04(2 \mathrm{H}, \mathrm{s}), 4.30-4.27(\mathrm{t}, J=6.61 \mathrm{~Hz}, 2 \mathrm{H}), 1.77-1.70(\mathrm{~m}, 2 \mathrm{H}), 1.52-$ $1.42(\mathrm{~m}, 2 \mathrm{H}), 1.00-0.96(\mathrm{t}, J=7.39 \mathrm{~Hz}, 3 \mathrm{H}) .{ }^{13} \mathrm{C} \mathrm{NMR}\left(100 \mathrm{MHz}, \mathrm{CDCl}_{3}\right) \delta: 166.26$, $151.69,147.88,125.45,124.76,109.70,108.15,101.98,65.00,31.01,19.51,14.01$. IR $\left(\mathrm{KBr}, \mathrm{cm}^{-1}\right): 2961,1713,1626,1608,1491,1443,1364,1258,1160,1105,1039,937$, 762. Anal. Calcd. for $\mathrm{C}_{12} \mathrm{H}_{14} \mathrm{O}_{4}$ : C, 64.85; H, 6.35. Found: C, 64.89; H, 6.42 . 


\section{butyl 2-methylbenzo $[d]$ thiazole-5-carboxylate}

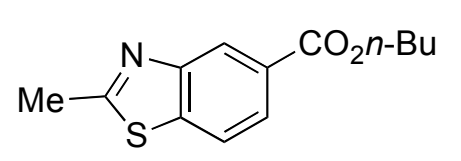

Following the general procedure, a mixture of 2methylbenzo $[d]$ thiazol-5-yl methanesulfonate $(1.0 \mathrm{mmol}$, $243 \mathrm{mg}$ ), $n$-butanol (3.06 mmol, 3.06 equiv., $0.28 \mathrm{~mL}$ ),

$\mathrm{Pd}(\mathrm{OAc})_{2}(2 \mathrm{~mol} \mathrm{\% ,} 0.02 \mathrm{mmol}, 0.02$ equiv., $4.5 \mathrm{mg})$, dcpp• $2 \mathrm{HBF}_{4}(2.2 \mathrm{~mol} \%, 0.022$ mmol, 0.022 equiv., $14.0 \mathrm{mg}), \mathrm{K}_{2} \mathrm{CO}_{3}(2.0 \mathrm{mmol}, 2.0$ equiv., $276 \mathrm{mg}), 4 \AA$ molecular sieves $(150 \mathrm{mg})$, and toluene $(1.0 \mathrm{~mL})$ was heated at $80{ }^{\circ} \mathrm{C}$ for $15 \mathrm{~h}$. The reaction mixture was filtered through Celite eluting with ethyl acetate and the solvent was removed in vacuo. The residue was purified by flash chromatography on silica gel $(20 \%$ EtOAc in hexanes) to provide the title compound as a white solid (203 mg, $82 \%$ ). mp 37-38 ${ }^{\circ} \mathrm{C} .{ }^{1} \mathrm{H}$ NMR $\left(400 \mathrm{MHz}, \mathrm{CDCl}_{3}\right) \delta: 8.58(\mathrm{~d}, J=1.53 \mathrm{~Hz}, 1 \mathrm{H}), 8.01-7.99(\mathrm{dd}, J=$ 8.38, $1.59 \mathrm{~Hz}, 1 \mathrm{H}), 7.85-7.82(\mathrm{~d}, J=8.38 \mathrm{~Hz}, 1 \mathrm{H}), 4.34-4.31(\mathrm{t}, J=6.53 \mathrm{~Hz}, 2 \mathrm{H}), 2.83$ (s, 3H), 1.77-1.70 (m, 2H), 1.51-1.42 (m, 2H), 0.97-0.94 (t, $J=7.39 \mathrm{~Hz}, 3 \mathrm{H}) .{ }^{13} \mathrm{C}$ NMR $\left(100 \mathrm{MHz}, \mathrm{CDCl}_{3}\right)$ 8: 168.62, 166.73, 153.33, 140.61, 128.79, 125.61, 123.97, 121.48, 65.28, 30.93, 20.55, 19.53, 14.04. IR (KBr, $\left.\mathrm{cm}^{-1}\right): 2959,2873,1716,1606,1529,1455$, 1417, 1297, 1247, 1172, 1093, 760. Anal. Calcd. for $\mathrm{C}_{13} \mathrm{H}_{15} \mathrm{NO}_{2} \mathrm{~S}: \mathrm{C}, 62.62 ; \mathrm{H}, 6.06$. Found: C, 62.89; H, 6.19 .

\section{butyl 4-(1H-pyrrol-1-yl)benzoate}

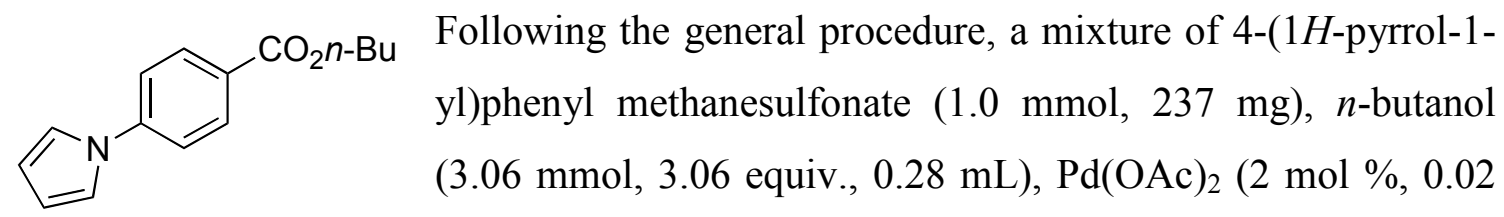

mmol, 0.02 equiv., $4.5 \mathrm{mg}$ ), dcpp• $2 \mathrm{HBF}_{4}(2.2 \mathrm{~mol} \%, 0.022 \mathrm{mmol}, 0.022$ equiv., 14.0 $\mathrm{mg}$ ), $\mathrm{K}_{2} \mathrm{CO}_{3}$ (2.0 mmol, 2.0 equiv., $276 \mathrm{mg}$ ), $4 \AA$ molecular sieves (150 mg), and toluene $(1.0 \mathrm{~mL})$ was heated at $100{ }^{\circ} \mathrm{C}$ for $15 \mathrm{~h}$. The reaction mixture was filtered through Celite eluting with ethyl acetate and the solvent was removed in vacuo. The residue was purified by flash chromatography on silica gel (20\% EtOAc in hexanes) to provide the title compound as a white solid (230 mg, $95 \%$ ). mp 61-62 ${ }^{\circ} \mathrm{C} .{ }^{1} \mathrm{H}$ NMR $(400 \mathrm{MHz}$, $\left.\mathrm{CDCl}_{3}\right) \delta:$ 8.10-8.07 (d, $\left.J=8.84 \mathrm{~Hz}, 2 \mathrm{H}\right), 7.44-7.40(\mathrm{~d}, J=8.81 \mathrm{~Hz}, 2 \mathrm{H}), 7.15-7.13(\mathrm{t}, J$ $=2.21 \mathrm{~Hz}, 2 \mathrm{H}), 6.37-6.36(\mathrm{t}, J=2.21 \mathrm{~Hz}, 2 \mathrm{H}), 4.34-4.31(\mathrm{t}, J=6.63 \mathrm{~Hz}, 2 \mathrm{H}), 1.79-1.71$ $(\mathrm{m}, 2 \mathrm{H}), 1.52-1.43(\mathrm{~m}, 2 \mathrm{H}), 1.00-0.96(\mathrm{t}, J=7.39 \mathrm{~Hz}, 3 \mathrm{H}) .{ }^{13} \mathrm{C}$ NMR $\left(100 \mathrm{MHz}, \mathrm{CDCl}_{3}\right)$ 
$\delta: 166.19,144.09,131.46,127.50,119.47,119.23,111.66,65.13,31.00,19.51,14.01$. IR $\left(\mathrm{KBr}, \mathrm{cm}^{-1}\right): 3145,2958,2931,2872,1704,1609,1522,1475,1334,1281,1182,1121$, 1068, 729. Anal. Calcd. for $\mathrm{C}_{15} \mathrm{H}_{17} \mathrm{NO}_{2}: \mathrm{C}, 74.05 ; \mathrm{H}, 7.04$. Found: C, 73.97; H, 7.07.

\section{butyl 4-ethanoyl-2-methoxybenzoate}<smiles>CCOC(=O)c1ccc(C(C)=O)cc1OC</smiles>

Following the general procedure, a mixture of 4-ethanoyl-2methoxyphenyl methanesulfonate (1.0 mmol, $244 \mathrm{mg}), n$ butanol (3.06 mmol, 3.06 equiv., $0.28 \mathrm{~mL}), \mathrm{Pd}(\mathrm{OAc})_{2}(2 \mathrm{~mol}$ \%, 0.02 mmol, 0.02 equiv., $4.5 \mathrm{mg})$, dcpp $2 \mathrm{HBF}_{4}(2.2 \mathrm{~mol} \%$, 0.022 mmol, 0.022 equiv., $14.0 \mathrm{mg}), \mathrm{K}_{2} \mathrm{CO}_{3}$ (2.0 mmol, 2.0 equiv., $\left.276 \mathrm{mg}\right), 4 \AA$ molecular sieves $(150 \mathrm{mg})$, and toluene $(1.0 \mathrm{~mL})$ was heated at $80{ }^{\circ} \mathrm{C}$ for $15 \mathrm{~h}$. The reaction mixture was filtered through Celite eluting with ethyl acetate and the solvent was removed in vacuo. The residue was purified by flash chromatography on silica gel $(20 \%$ EtOAc in hexanes) to provide the title compound as a colorless oil (236 mg, $94 \%) .{ }^{1} \mathrm{H}$

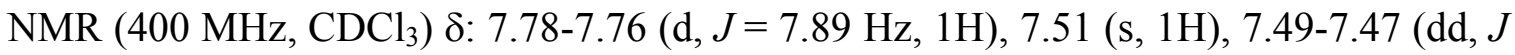
$=7.92,1.43 \mathrm{~Hz}, 1 \mathrm{H}), 4.29-4.26(\mathrm{t}, J=6.61 \mathrm{~Hz}, 2 \mathrm{H}), 3.91(\mathrm{~s}, 3 \mathrm{H}), 2.58(\mathrm{~s}, 3 \mathrm{H}), 1.73-1.66$ $(\mathrm{m}, 2 \mathrm{H}), 1.48-1.38(\mathrm{~m}, 2 \mathrm{H}), 0.95-0.91(\mathrm{t}, J=7.39 \mathrm{~Hz}, 3 \mathrm{H}) .{ }^{13} \mathrm{C} \mathrm{NMR}\left(100 \mathrm{MHz}, \mathrm{CDCl}_{3}\right)$ $\delta: 197.58,165.98,159.10,140.82,131.67,125.04,120.61,110.91,65.33,56.32,30.84$, 27.04, 19.39, 13.92. IR ( $\left.\mathrm{KBr}, \mathrm{cm}^{-1}\right): 2961,2874,1731,1690,1572,1409,1290,1225$, 1098, 1073, 1032. Anal. Calcd. for $\mathrm{C}_{14} \mathrm{H}_{18} \mathrm{O}_{4}$ : C, 67.18; H, 7.25. Found: C, 67.51; H, 7.26 .

\section{butyl 5-formyl-2-methoxybenzoate}<smiles>CCCCC(=O)c1cc(C=O)ccc1OC</smiles>

Following the general procedure, a mixture of 5-formyl-2methoxyphenyl 4-methylbenzenesulfonate $(1.0 \mathrm{mmol}, 306 \mathrm{mg})$, $n$-butanol (3.06 mmol, 3.06 equiv., $0.28 \mathrm{~mL}), \mathrm{Pd}(\mathrm{OAc})_{2}(2 \mathrm{~mol}$

\%, 0.02 mmol, 0.02 equiv., $4.5 \mathrm{mg}$ ), dcpp• $2 \mathrm{HBF}_{4}$ (2.2 mol \%, $0.022 \mathrm{mmol}, 0.022$ equiv., $14.0 \mathrm{mg}), \mathrm{K}_{2} \mathrm{CO}_{3}$ (2.0 mmol, 2.0 equiv., $276 \mathrm{mg}$ ), $4 \AA$ molecular sieves (150 mg), and toluene $(1.0 \mathrm{~mL})$ was heated at $80{ }^{\circ} \mathrm{C}$ for $15 \mathrm{~h}$. The reaction mixture was filtered through Celite eluting with ethyl acetate and the solvent was removed in vacuo. The residue was purified by flash chromatography on silica gel (20\% EtOAc in hexanes) to provide the 
title compound as a colorless oil (223 mg, $95 \%)$. ${ }^{1} \mathrm{H}$ NMR (400 MHz, $\left.\mathrm{CDCl}_{3}\right) \delta: 9.87(\mathrm{~s}$, $1 \mathrm{H}), 8.26-8.25(\mathrm{~d}, J=2.19 \mathrm{~Hz}, 1 \mathrm{H}), 7.98-7.95(\mathrm{dd}, J=8.68,2.20 \mathrm{~Hz}, 1 \mathrm{H}), 7.07-7.05(\mathrm{~d}, J$ $=8.69 \mathrm{~Hz}, 1 \mathrm{H}), 4.30-4.27(\mathrm{t}, J=6.65 \mathrm{~Hz}, 2 \mathrm{H}), 3.95(\mathrm{~s}, 3 \mathrm{H}), 1.74-1.67(\mathrm{~m}, 2 \mathrm{H}), 1.48-1.39$ (m, 2H), 0.95-0.93 (t, $J=7.39 \mathrm{~Hz}, 3 \mathrm{H}) .{ }^{13} \mathrm{C} \mathrm{NMR}\left(100 \mathrm{MHz}, \mathrm{CDCl}_{3}\right) \delta: 190.34,165.34$, $163.80,134.61,134.29,129.21,121.22,112.42,65.35,56.61,30.86,19.41,13.93$. IR $\left(\mathrm{KBr}, \mathrm{cm}^{-1}\right): 2961,1699,1603,1500,1274,1017,822$. Anal. Calcd. for $\mathrm{C}_{13} \mathrm{H}_{16} \mathrm{O}_{4}$ : C, 66.09; H, 6.83. Found: C, 66.15; H, 6.91.

\section{butyl 2,4-dimethylbenzoate}

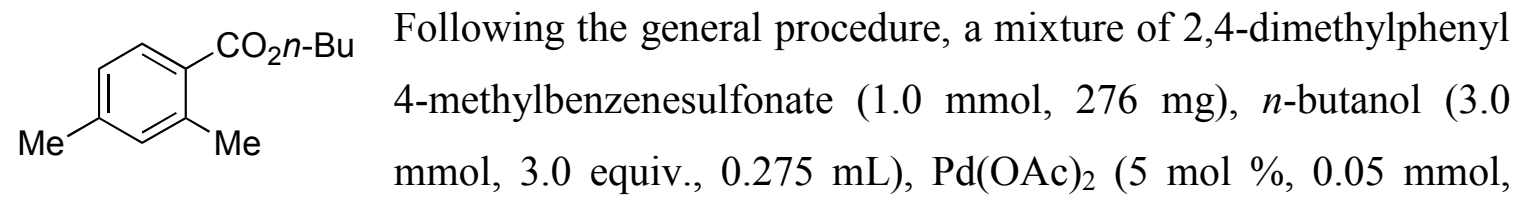

0.05 equiv., $11.2 \mathrm{mg})$, dcpp $2 \mathrm{HBF}_{4}$ (6 mol \%, $0.06 \mathrm{mmol}, 0.06$ equiv., $\left.36.7 \mathrm{mg}\right), \mathrm{K}_{2} \mathrm{CO}_{3}$ (2.0 mmol, 2.0 equiv., $276 \mathrm{mg}), 4 \AA$ molecular sieves $(150 \mathrm{mg})$, and toluene $(1.5 \mathrm{~mL})$ was heated at $100{ }^{\circ} \mathrm{C}$ for $22 \mathrm{~h}$. The reaction mixture was filtered through Celite eluting with ethyl acetate and the solvent was removed in vacuo. The reaction was judged to have proceeded to $88 \%$ conversion of the starting material by GC analysis. This residue was purified by flash chromatography on silica gel (5\% EtOAc in hexanes) to provide the title compound as a colorless oil (163 mg, $79 \%):{ }^{1} \mathrm{H} \mathrm{NMR}\left(300 \mathrm{MHz}, \mathrm{CDCl}_{3}\right)$ 8: 7.90-7.78 (m, 1H), 7.14-6.98 (m, 2H), $4.29(\mathrm{t}, J=6.59 \mathrm{~Hz}, 2 \mathrm{H}), 2.58(\mathrm{~s}, 3 \mathrm{H}), 2.36(\mathrm{~s}, 3 \mathrm{H}), 1.83-$ $1.68(\mathrm{~m}, 2 \mathrm{H}), 1.57-1.39(\mathrm{~m}, 2 \mathrm{H}), 7.38(\mathrm{t}, J=7.38 \mathrm{~Hz}, 3 \mathrm{H}) .{ }^{13} \mathrm{C} \mathrm{NMR}\left(75 \mathrm{MHz}, \mathrm{CDCl}_{3}\right)$ $\delta: 167.8,142.5,140.4,132.6,130.9,127.1,126.6,64.5,31.0,22.0,21.5,19.6,13.9$. IR $\left(\mathrm{KBr}, \mathrm{cm}^{-1}\right): 2961,2932,2874,1718,1614,1572,1452,1381,1287,1258,1234,1167$, 1153, 1084, 1036, 965, 867, 834, 771, 724, 694.

\section{butyl 3-(trifluoromethyl)benzoate}

$$
\begin{aligned}
& \mathrm{CO}_{2} n-\mathrm{Bu} \\
& \begin{array}{l}
\text { Following the general procedure, a mixture of 3- } \\
\text { (trifluoromethyl)phenyl methanesulfonate }(1.0 \mathrm{mmol}, 240 \mathrm{mg}) \text {, } \\
n \text {-butanol }(3.06 \mathrm{mmol}, 3.06 \text { equiv., } 0.28 \mathrm{~mL}), \mathrm{Pd}(\mathrm{OAc})_{2}(2 \mathrm{~mol}
\end{array}
\end{aligned}
$$

\%, 0.02 mmol, 0.02 equiv., $4.5 \mathrm{mg}$ ), dcpp $2 \mathrm{HBF}_{4}$ (2.2 mol \%, $0.022 \mathrm{mmol}, 0.022$ equiv., $14.0 \mathrm{mg}), \mathrm{K}_{2} \mathrm{CO}_{3}$ (2.0 mmol, 2.0 equiv., $276 \mathrm{mg}$ ), $4 \AA$ molecular sieves (150 mg), and 
toluene $(1.0 \mathrm{~mL})$ was heated at $80{ }^{\circ} \mathrm{C}$ for $15 \mathrm{~h}$. The reaction mixture was filtered through Celite eluting with ethyl acetate and the solvent was removed in vacuo. The residue was purified by flash chromatography on silica gel (10\% EtOAc in hexanes) to provide the title compound as a colorless oil (214 mg, 87\%). ${ }^{1} \mathrm{H}$ NMR (400 MHz, $\left.\mathrm{CDCl}_{3}\right)$ 8: 8.27 (s, $1 \mathrm{H}), 8.21-8.19(\mathrm{~d}, J=7.82 \mathrm{~Hz}, 1 \mathrm{H}), 7.79-7.77(\mathrm{~d}, J=7.81 \mathrm{~Hz}, 1 \mathrm{H}), 7.57-7.53(\mathrm{t}, J=7.82$ $\mathrm{Hz}, 1 \mathrm{H}), 4.35-4.33$ (t, $J=6.67 \mathrm{~Hz}, 2 \mathrm{H}), 1.78-1.71(\mathrm{~m}, 2 \mathrm{H}), 1.50-1.40$ (m, 2H), 0.98-0.94 (t, $J=7.40 \mathrm{~Hz}, 3 \mathrm{H}) .{ }^{13} \mathrm{C}$ NMR $\left(100 \mathrm{MHz}, \mathrm{CDCl}_{3}\right) \delta: 165.54,132.94,131.53,131.18$ (q, $J_{\mathrm{CF}}=32.9 \mathrm{~Hz}$, chemical shifts for 4 peaks $\left.131.67,131.35,131.02,130.69\right), 129.52\left(\mathrm{q}, J_{\mathrm{CF}}\right.$ $=3.6 \mathrm{~Hz}$, chemical shifts for 4 peaks $129.57,129.54,129.50,129.46), 129.19,126.64(\mathrm{q}$, $J_{\mathrm{CF}}=3.8 \mathrm{~Hz}$, chemical shifts for 4 peaks $\left.126.70,126.66,126.62,126.58\right), 123.88$ (q, $J_{\mathrm{CF}}$ $=272.4 \mathrm{~Hz}$, chemical shifts for 4 peaks 127.94, 125.23, 122.52, 119.81), 65.62, 30.87, 19.42, 13.93. IR (KBr, $\left.\mathrm{cm}^{-1}\right): 2964,1727,1336,1254,1170,1132,1073,758,695$. Anal. Calcd. for $\mathrm{C}_{12} \mathrm{H}_{13} \mathrm{~F}_{3} \mathrm{O}_{2}$ : C, 58.54; H, 5.32. Found: C, 58.50; H, 5.45.

\section{ethyl 3-(trifluoromethyl)benzoate ${ }^{14}$}

$$
\begin{aligned}
& \text { Following the general procedure, a mixture of 3- } \\
& \text { (trifluoromethyl)phenyl 4-methylbenzenesulfonate }(1.0 \mathrm{mmol}, 316 \\
& \mathrm{mg}) \text {, ethanol }(10 \mathrm{mmol}, 10 \text { equiv., } 0.58 \mathrm{~mL}), \mathrm{Pd}(\mathrm{OAc})_{2}(2 \mathrm{~mol} \% \text {, }
\end{aligned}
$$
$0.02 \mathrm{mmol}, 0.02$ equiv., $4.5 \mathrm{mg})$, dcpp• $2 \mathrm{HBF}_{4}(2.2 \mathrm{~mol} \%, 0.022 \mathrm{mmol}, 0.022$ equiv., $14.0 \mathrm{mg}), \mathrm{K}_{2} \mathrm{CO}_{3}(2.0 \mathrm{mmol}, 2.0$ equiv., $276 \mathrm{mg}), 4 \AA$ molecular sieves $(150 \mathrm{mg})$, and toluene $(1.0 \mathrm{~mL})$ was heated at $80{ }^{\circ} \mathrm{C}$ for $15 \mathrm{~h}$. The reaction mixture was filtered through Celite eluting with ethyl acetate and the solvent was removed in vacuo. The residue was purified by flash chromatography on silica gel (20\% EtOAc in hexanes) to provide the title compound as a colorless oil (190 mg, $87 \%$ ). ${ }^{1} \mathrm{H}$ NMR (400 MHz, $\left.\mathrm{CDCl}_{3}\right) \delta: 8.28$ (s, $1 \mathrm{H}), 8.21-8.19(\mathrm{~d}, J=7.81 \mathrm{~Hz}, 1 \mathrm{H}), 7.79-7.77(\mathrm{~d}, J=7.81 \mathrm{~Hz}, 1 \mathrm{H}), 7.57-7.53(\mathrm{t}, J=7.82$ $\mathrm{Hz}, 1 \mathrm{H}), 4.42-4.36$ (q, $J=7.14 \mathrm{~Hz}, 2 \mathrm{H}), 1.41-1.37$ (t, $J=7.14 \mathrm{~Hz}, 3 \mathrm{H}) .{ }^{13} \mathrm{C}$ NMR $(100$ $\mathrm{MHz}, \mathrm{CDCl}_{3}$ ) $\delta: 165.48,132.96,131.50,131.17$ (q, $J=32.94 \mathrm{~Hz}$, chemical shifts for 4 peaks: $131.65,131.33,131.00,130.67), 129.52(\mathrm{q}, J=3.64 \mathrm{~Hz}$, chemical shifts for 4 peaks: $129.58,129.54,129.50,129.47), 129.18,126.66$ (q, $J=3.84 \mathrm{~Hz}$, chemical shifts of 4 peaks: $126.72,126.68,126.64,126.60), 123.88$ (q, $J=272.41 \mathrm{~Hz}$, chemical shifts of 4 peaks: $127.94,125.23,122.53,119.82), 61.75,14.47 . \mathrm{IR}\left(\mathrm{KBr}, \mathrm{cm}^{-1}\right): 2987,1728$, 
1618, 1445, 1371, 1336, 1256, 1171, 1131, 1073, 1022, 758, 695. Anal. Calcd. for $\mathrm{C}_{10} \mathrm{H}_{9} \mathrm{~F}_{3} \mathrm{O}_{2}$ : C, 55.05; H, 4.16. Found: $\mathrm{C}, 55.30 ; \mathrm{H}, 4.39$.

\section{cyclopropylmethyl 2-methylquinoline-8-carboxylate}

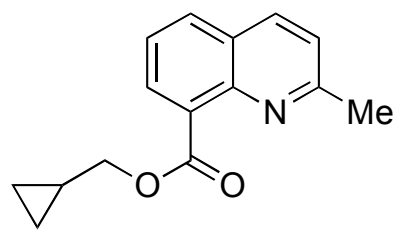

Following the general procedure, a mixture of

2-methylquinolin-8-yl 4-methylbenzenesulfonate $(1.0 \mathrm{mmol}$,

$313 \mathrm{mg}$ ), cyclopropyl carbinol (3.00 mmol, 3.00 equiv., 243

$\mu \mathrm{L}), \mathrm{Pd}(\mathrm{OAc})_{2}(2 \mathrm{~mol} \%, 0.02 \mathrm{mmol}, 0.02$ equiv., $4.5 \mathrm{mg})$,

dcpp• $2 \mathrm{HBF}_{4}(2.2 \mathrm{~mol} \%, 0.022 \mathrm{mmol}, 0.022$ equiv., $14.0 \mathrm{mg}), \mathrm{K}_{2} \mathrm{CO}_{3}(2.0 \mathrm{mmol}, 2.0$ equiv., $276 \mathrm{mg}), 4 \AA$ molecular sieves $(150 \mathrm{mg})$, and toluene $(1.0 \mathrm{~mL})$ was heated at 90 ${ }^{\circ} \mathrm{C}$ for $15 \mathrm{~h}$. The reaction mixture was filtered through Celite eluting with ethyl acetate and the solvent was removed in vacuo. The residue was purified by flash chromatography on silica gel (20\% EtOAc in hexanes) to provide the title compound as a pale yellow solid (226 mg, 94 \%). mp 58-60 ${ }^{\circ} \mathrm{C} .{ }^{1} \mathrm{H}$ NMR $\left(400 \mathrm{MHz}, \mathrm{CDCl}_{3}\right)$ 8: 8.01-7.99 (d, J=8.44 $\mathrm{Hz}, 1 \mathrm{H}), 7.95-7.93$ (dd, $J=7.16,1.46 \mathrm{~Hz}, 1 \mathrm{H}), 7.85-7.82$ (dd, $J=8.13,1.33 \mathrm{~Hz}, 1 \mathrm{H}$ ), 7.47- 7.44 (t, $J=7.65 \mathrm{~Hz}, 1 \mathrm{H}), 7.29-7.27$ (d, $J=8.43 \mathrm{~Hz}, 1 \mathrm{H}), 4.29-4.27$ (d, $J=7.22$ $\mathrm{Hz}, 2 \mathrm{H}), 2.73$ (s, 3H), 1.33-1.29 (m, 1H), 0.64-0.59 (m, 2H), 0.44-0.40 (m, 2H). ${ }^{13} \mathrm{C}$ NMR $\left(100 \mathrm{MHz}, \mathrm{CDCl}_{3}\right) \delta: 168.62,160.44,145.43,136.18,131.81,130.84,129.99$, 126.81, 124.84, 122.69, 70.26, 26.06, 10.12, 3.62. IR ( $\left.\mathrm{KBr}, \mathrm{cm}^{-1}\right): 3007,2950,1727$, $1614,1602,1571,1500,1275,1194,1144,1037$. Anal. Calcd. for $\mathrm{C}_{15} \mathrm{H}_{15} \mathrm{NO}_{2}$ : C, 74.67; H, 6.27. Found: C, 74.75; H, 6.29.

\section{(2,2-dimethyl-1,3-dioxolan-4-yl)methyl 3,4,5-trimethoxybenzoate}

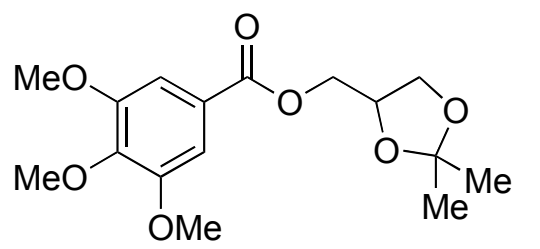

Following the general procedure, a mixture of 3,4,5trimethoxyphenyl methanesulfonate $(1.0 \mathrm{mmol}, 262$ $\mathrm{mg}$ ), solketal (3.00 mmol, 3.00 equiv., $373 \mu \mathrm{l})$, $\operatorname{Pd}(\mathrm{OAc})_{2}(2 \mathrm{~mol} \%, 0.02 \mathrm{mmol}, 0.02$ equiv., $4.5 \mathrm{mg})$, dcpp•2 $2 \mathrm{HBF}_{4}(2.2 \mathrm{~mol} \%, 0.022 \mathrm{mmol}, 0.022$ equiv., $14.0 \mathrm{mg}), \mathrm{K}_{2} \mathrm{CO}_{3}(2.0 \mathrm{mmol}, 2.0$ equiv., $276 \mathrm{mg}), 4 \AA$ molecular sieves $(150 \mathrm{mg})$, and toluene $(1.0 \mathrm{~mL})$ was heated at 90 ${ }^{\circ} \mathrm{C}$ for $15 \mathrm{~h}$. The reaction mixture was filtered through Celite eluting with ethyl acetate and the solvent was removed in vacuo. The residue was purified by flash chromatography 
on silica gel (20\% EtOAc in hexanes) to provide the title compound as a pale yellow oil (282 mg, 87 \%). ${ }^{1} \mathrm{H}$ NMR (400 MHz, $\mathrm{CDCl}_{3}$ ) $\delta: 7.28$ (s, 2H), 4.45-4.40 (m, 1H), 4.354.34 (m, 2H), 4.13-4.09 (dd, $J=8.45,6.49 \mathrm{~Hz}, 1 \mathrm{H}), 3.90-3.82(\mathrm{~m}, 1 \mathrm{H}), 3.87(\mathrm{~s}, 9 \mathrm{H}), 1.43$ (s, 3H), 1.35 (s, 3H). ${ }^{13} \mathrm{C}$ NMR (100 MHz, $\left.\mathrm{CDCl}_{3}\right) \delta: 166.23,153.12,142.52,124.95$, 110.03, 107.09, 73.90, 66.47, 65.26, 61.13, 56.42, 26.90, 25.55. IR (KBr, $\left.\mathrm{cm}^{-1}\right): 2942$, 1718, 1590, 1504, 1462, 1416, 1334, 1129, 1004, 842, 762. Anal. Calcd. for $\mathrm{C}_{16} \mathrm{H}_{22} \mathrm{O}_{7}$ : C, 58.89; H, 6.79. Found: C, 58.82; H, 6.90.

\section{neopentyl 3-cyanobenzoate}<smiles>CC(C)(C)COC(=O)c1cccc(C#N)c1</smiles>

Following the general procedure, a mixture of 3-cyanophenyl 4-methylbenzenesulfonate $(1.0 \mathrm{mmol}, 273 \mathrm{mg})$, neopentyl alcohol (3.00 mmol, 3.00 equiv., $264 \mathrm{mg}), \operatorname{Pd}(\mathrm{OAc})_{2}(2 \mathrm{~mol} \%$, $0.02 \mathrm{mmol}, 0.02$ equiv., $4.5 \mathrm{mg})$, dcpp• $2 \mathrm{HBF}_{4}(2.2 \mathrm{~mol} \%, 0.022 \mathrm{mmol}, 0.022$ equiv., $14.0 \mathrm{mg}), \mathrm{K}_{2} \mathrm{CO}_{3}(2.0 \mathrm{mmol}, 2.0$ equiv., $276 \mathrm{mg}), 4 \AA$ molecular sieves $(150 \mathrm{mg})$, and toluene $(1.0 \mathrm{~mL})$ was heated at $90{ }^{\circ} \mathrm{C}$ for $15 \mathrm{~h}$. The reaction mixture was filtered through Celite eluting with ethyl acetate and the solvent was removed in vacuo. The residue was purified by flash chromatography on silica gel ( $20 \%$ EtOAc in hexanes) to provide the title compound as a colorless oil (199 mg, $92 \%$ ). ${ }^{1} \mathrm{H}$ NMR (400 MHz, $\mathrm{CDCl}_{3}$ ) $\delta: 8.33$ (s, $1 \mathrm{H}), 8.30-8.28$ (dt, $J=8.02,1.43 \mathrm{~Hz}, 1 \mathrm{H}), 7.87-7.84$ (dt, $J=7.77,1.42 \mathrm{~Hz}, 1 \mathrm{H}), 7.63-$ $7.59(\mathrm{t}, J=7.83 \mathrm{~Hz}, 1 \mathrm{H}), 4.06(\mathrm{~s}, 2 \mathrm{H}), 1.06(\mathrm{~s}, 9 \mathrm{H}) .{ }^{13} \mathrm{C} \mathrm{NMR}\left(100 \mathrm{MHz}, \mathrm{CDCl}_{3}\right) \delta$ : $164.83,136.15,133.87,133.38,132.01,129.70,118.22,113.18,75.21,31.85,26.77$. IR $\left(\mathrm{KBr}, \mathrm{cm}^{-1}\right):$ 2961, 2234, 1727, 1605, 1584, 1479, 1371, 1286, 1187, 1107, 979, 753, 681. Anal. Calcd. for $\mathrm{C}_{13} \mathrm{H}_{15} \mathrm{NO}_{2}$ : C, 71.87; H, 6.96. Found: C, 72.11; H, 7.12.

\section{cyclopentyl biphenyl-4-carboxylate}

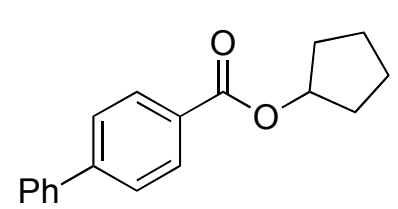

Following the general procedure, a mixture of biphenyl-4-yl methanesulfonate $(1.0 \mathrm{mmol}, 248 \mathrm{mg})$, cyclopentanol (3.00 mmol, 3.00 equiv., $272 \mu \mathrm{l}), \mathrm{Pd}(\mathrm{OAc})_{2}(2 \mathrm{~mol} \%, 0.02 \mathrm{mmol}$, 0.02 equiv., $4.5 \mathrm{mg})$, dcpp $2 \mathrm{HBF}_{4}(2.2 \mathrm{~mol} \%, 0.022 \mathrm{mmol}, 0.022$ equiv., $14.0 \mathrm{mg})$, $\mathrm{K}_{2} \mathrm{CO}_{3}$ (2.0 mmol, 2.0 equiv., $\left.276 \mathrm{mg}\right), 4 \AA$ molecular sieves $(150 \mathrm{mg}$ ), and toluene (1.0 $\mathrm{mL}$ ) was heated at $110{ }^{\circ} \mathrm{C}$ for $15 \mathrm{~h}$. The reaction mixture was filtered through Celite 
eluting with ethyl acetate and the solvent was removed in vacuo. The residue was purified by flash chromatography on silica gel (10\% EtOAc in hexanes) to provide the title compound as a colorless oil (204 mg, $77 \%$ ). ${ }^{1} \mathrm{H}$ NMR (400 MHz, $\left.\mathrm{CDCl}_{3}\right)$ 8: 8.08$8.06(\mathrm{~d}, J=8.50 \mathrm{~Hz}, 2 \mathrm{H}), 7.64-7.59(\mathrm{~m}, 4 \mathrm{H}), 7.46-7.43(\mathrm{t}, J=7.43 \mathrm{~Hz}, 2 \mathrm{H}), 7.39-7.35$ $(\mathrm{m}, 1 \mathrm{H}), 5.43-5.39(\mathrm{~m}, 1 \mathrm{H}), 1.99-1.91(\mathrm{~m}, 2 \mathrm{H}), 1.86-1.79(\mathrm{~m}, 4 \mathrm{H}), 1.70-1.63(\mathrm{~m}, 2 \mathrm{H}) .{ }^{13} \mathrm{C}$ NMR $\left(100 \mathrm{MHz}, \mathrm{CDCl}_{3}\right) \delta: 166.46,145.61,140.30,130.21,129.83,129.12,128.28$, 127.48, 127.17, 77.91, 33.02, 24.05. IR (KBr, $\left.\mathrm{cm}^{-1}\right): 3059,3031,2964,2872,1710,1609$, 1487, 1449, 1405, 1278, 1177, 1114, 961, 859, 747, 697. Anal. Calcd. for $\mathrm{C}_{18} \mathrm{H}_{18} \mathrm{O}_{2}: \mathrm{C}$, 81.17; H, 6.81. Found: C, 81.11; H, 6.83 .

\section{ethyl 3-(dimethylamino)benzoate}

$$
\begin{aligned}
& \mathrm{Me}_{2} \mathrm{~N} \\
& \mathrm{CO}_{2} \mathrm{Et} \\
& \begin{array}{l}
\text { Following the general procedure, a mixture of 3- } \\
\text { (dimethylamino }) \text { phenyl methanesulfonate }(1.0 \mathrm{mmol}, 215 \mathrm{mg}) \text {, } \\
\text { ethanol }(5 \mathrm{mmol}, 5 \text { equiv., } 0.29 \mathrm{~mL}), \mathrm{Pd}(\mathrm{OAc})_{2}(4 \mathrm{~mol} \%, 0.04
\end{array}
\end{aligned}
$$

mmol, 0.04 equiv., $9 \mathrm{mg}$ ), dcpp•2 $2 \mathrm{HBF}_{4}(4.4 \mathrm{~mol} \%, 0.044 \mathrm{mmol}, 0.044$ equiv., $27.0 \mathrm{mg}$ ), $\mathrm{K}_{2} \mathrm{CO}_{3}$ (2.0 mmol, 2.0 equiv., $\left.276 \mathrm{mg}\right), 4 \AA$ molecular sieves $(150 \mathrm{mg})$, and toluene (1.0 $\mathrm{mL}$ ) was heated at $80{ }^{\circ} \mathrm{C}$ for $20 \mathrm{~h}$. The reaction mixture was filtered through Celite eluting with ethyl acetate and the solvent was removed in vacuo. The residue was purified by flash chromatography on silica gel (gradient elution: $0 \%$ to $10 \%$ EtOAc in hexanes) to provide the title compound as a colorless oil (171 mg, $88 \%$ ). ${ }^{1} \mathrm{H}$ NMR (300 $\left.\mathrm{MHz}, \mathrm{CDCl}_{3}\right)$ 8: 7.39-7.33 (m, 2H), 7.28-7.21 (m, 1H), 6.89-6.83 (m, 1H), $4.33(\mathrm{q}, J=$ $7.14 \mathrm{~Hz}, 2 \mathrm{H}), 2.95$ (s, 6H) $1.35(\mathrm{t}, J=7.14 \mathrm{~Hz}, 3 \mathrm{H}) .{ }^{13} \mathrm{C} \mathrm{NMR}\left(75 \mathrm{MHz}, \mathrm{CDCl}_{3}\right) \delta$ : 167.26, 150.40, 131.09, 128.95, 117.44, 116.62, 113.10, 60.79, 40.46, 14.37. IR (KBr, $\mathrm{cm}^{-1}$ ): 2981, 2903, 2807, 1716, 1603, 1578, 1498, 1438, 1367, 1353, 1308, 1263, 1232, 1182, 1110, 1083, 1063, 1027, 995, 965, 867, 799, 753, 684, 667. Anal. Calcd. for $\mathrm{C}_{11} \mathrm{H}_{15} \mathrm{NO}_{2}: \mathrm{C}, 68.37 ; \mathrm{H}, 7.82$. Found: $\mathrm{C}, 68.61 ; \mathrm{H}, 7.97$.

\section{ethyl 4-methoxybenzoate ${ }^{15}$}

$$
\begin{aligned}
& \mathrm{CO}_{2} \mathrm{Et} \\
& \begin{array}{l}
\text { Following the general procedure, a mixture of 4-methoxyphenyl 4- } \\
\text { fluorobenzenesulfonate }(1.0 \mathrm{mmol}, 282 \mathrm{mg}) \text {, ethanol }(10 \mathrm{mmol}, 10 \\
\text { equiv., } 0.58 \mathrm{~mL}), \mathrm{Pd}(\mathrm{OAc})_{2}(4 \mathrm{~mol} \%, 0.04 \mathrm{mmol}, 0.04 \text { equiv., } 9.0
\end{array}
\end{aligned}
$$


mg), dcpp $2 \mathrm{HBF}_{4}(4.4 \mathrm{~mol} \%, 0.044 \mathrm{mmol}, 0.044$ equiv., $28.0 \mathrm{mg}), \mathrm{K}_{2} \mathrm{CO}_{3}(2.0 \mathrm{mmol}$, 2.0 equiv., $276 \mathrm{mg}), 4 \AA$ molecular sieves $(150 \mathrm{mg})$, and toluene $(1.5 \mathrm{~mL})$ was heated at $80{ }^{\circ} \mathrm{C}$ for $15 \mathrm{~h}$. The reaction mixture was filtered through Celite eluting with ethyl acetate and the solvent was removed in vacuo. The residue was purified by flash chromatography on silica gel (10\% EtOAc in hexanes) to provide the title compound as a colorless oil (150 mg, $83 \%$ ). ${ }^{1} \mathrm{H}$ NMR (400 MHz, $\left.\mathrm{CDCl}_{3}\right) \delta: 7.98-7.96(\mathrm{~d}, J=8.90 \mathrm{~Hz}$, 2H), 6.89-6.87 (d, $J=8.84 \mathrm{~Hz}, 2 \mathrm{H}), 4.34-4.29$ (q, $J=7.13 \mathrm{~Hz}, 2 \mathrm{H}), 3.82$ (s, 3H), 1.36$1.33(\mathrm{t}, J=7.13 \mathrm{~Hz}, 3 \mathrm{H}) .{ }^{13} \mathrm{C} \mathrm{NMR}\left(100 \mathrm{MHz}, \mathrm{CDCl}_{3}\right) \delta: 166.59,163.42,131.72$, 123.12, 113.72, 60.83, 55.60, 14.58. IR (KBr, cm $\left.{ }^{-1}\right): 2981,2937,2841,1712,1607,1512$, 1464, 1367, 1316, 1257, 1169, 1103, 1031, 848, 772. Anal. Calcd. for $\mathrm{C}_{10} \mathrm{H}_{12} \mathrm{O}_{3}$ : C, 66.65; H, 6.71. Found: C, 66.36; H, 6.74.

\section{ethyl 5,6,7,8-tetrahydronaphthalene-2-carboxylate}

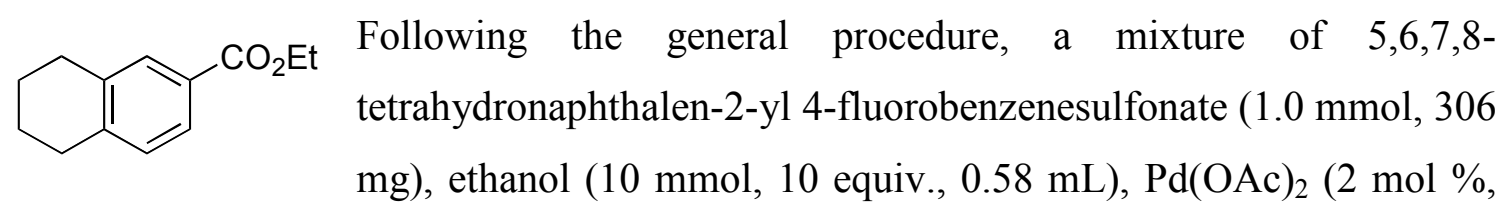
$0.02 \mathrm{mmol}, 0.02$ equiv., $4.5 \mathrm{mg})$, dcpp $2 \mathrm{HBF}_{4}(2.2 \mathrm{~mol} \%, 0.022 \mathrm{mmol}, 0.022$ equiv., $14.0 \mathrm{mg}), \mathrm{K}_{2} \mathrm{CO}_{3}$ (2.0 mmol, 2.0 equiv., $276 \mathrm{mg}$ ), $4 \AA$ molecular sieves (150 mg), and toluene $(1.0 \mathrm{~mL})$ was heated at $80{ }^{\circ} \mathrm{C}$ for $15 \mathrm{~h}$. The reaction mixture was filtered through Celite eluting with ethyl acetate and the solvent was removed in vacuo. The residue was purified by flash chromatography on silica gel (gradient elution: hexanes to $10 \%$ EtOAc in hexanes) to provide the title compound as a colorless oil $(173 \mathrm{mg}, 85 \%) .{ }^{1} \mathrm{H} \mathrm{NMR}$ $\left(400 \mathrm{MHz}, \mathrm{CDCl}_{3}\right) \delta: 7.72-7.70(\mathrm{~m}, 2 \mathrm{H}), 7.09-7.07(\mathrm{~d}, J=7.67 \mathrm{~Hz}, 1 \mathrm{H}), 4.35-4.30(\mathrm{q}, J=$ $7.07 \mathrm{~Hz}, 2 \mathrm{H}), 2.78(\mathrm{~m}, 4 \mathrm{H}), 1.80-1.76(\mathrm{~m}, 4 \mathrm{H}), 1.37-1.34(\mathrm{t}, J=7.06 \mathrm{~Hz}, 3 \mathrm{H}) .{ }^{13} \mathrm{C} \mathrm{NMR}$ $\left(100 \mathrm{MHz}, \mathrm{CDCl}_{3}\right) \delta: 167.14,142.87,137.40,130.53,129.30,127.81,126.65,60.88$, 29.79, 29.47, 23.16, 23.06, 14.57. IR (KBr, $\left.\mathrm{cm}^{-1}\right): 2981,2934,2860,1717,1612,1575$, 1420, 1366, 1274, 1232, 1181, 1120, 1100, 1024, 760. Anal. Calcd. for $\mathrm{C}_{13} \mathrm{H}_{16} \mathrm{O}_{2}$ : C, 76.44; H, 7.90. Found: C, 76.15; H, 7.99. 


\section{Experimental References}

G. Lin, A. Zhang, Tetrahedron, 2000, 56, 7163.

T. Ritter, K. Stanek, I. Larrosa, E. M. Carreira, Org. Lett., 2004, 6, 1513.

D. Zim, V. R. Lando, J. Dupont, A. L. Monteiro, Org. Lett., 2001, 3, 3049

J. C. Carnahan, Jr., W. D. Closson, J. R. Ganson, D. A. Juckett, K. S. Quaal, J. Am. Chem. Soc., 1976, 2526.

5 R. C. Huston, T. Y. Hsieh, J. Am. Chem. Soc., 1936, 58, 439.

6 M. R. del Guidice, G. Settmj, M. Delfini, Tetrahedron, 1984, 40, 4067

7 D. Collado, E. Perez-Inestrosa, R. Suau, J. Org. Chem., 2003, 68, 3574.

8 H. Rottendorf, S. Sternhell, Aust. J. Chem., 1963, 647.

9 Z.-Y. Tang, Q.-S. Hu, J. Am. Chem. Soc., 2004, 126, 3058.

10 A. Mori, T. Mizusaki, T. Ikawa, T. Maegawa, Y. Monguchi, H. Sajiki, Tetrahedron, 2007, 63, 1270

11 V. Percec, J.-Y. Bae, M. Zhao, D. H. Hill, J. Org. Chem., 1995, 60, 176.

12 W. Mägerlein, A. F. Indolese, M. Beller, J. Organometal. Chem., 2002, 641, 30.

13 H. Neumann, A. Brennführer, P. Groß, T. Riermeier, J. Almena, M. Beller, Adv. Synth. Catal., 2006, 348, 1255.

14 M. A. Al-Aseer, B. D. Allison, S. G. Smith, J. Org. Chem., 1985, 50, 2715.

15 C. Cai, N. R. Rivera, J. Balsells, R. R. Sidler, J. C. McWilliams, C. S. Schultz, Y. Sun, Org. Lett., 2006, 8, 5161. 